\title{
Practical aspects of Kelvin-probe force microscopy at solid/liquid interfaces in various liquid media
}

\section{$\operatorname{AUTHOR}(S)$ :}

Umeda, Ken-ichi; Kobayashi, Kei; Oyabu, Noriaki; Hirata, Yoshiki; Matsushige, Kazumi; Yamada, Hirofumi

\section{CITATION:}

Umeda, Ken-ichi ...[et al]. Practical aspects of Kelvin-probe force microscopy at solid/liquid interfaces in various liquid media. Journal of Applied Physics 2014, 116(13): 134307.

\section{ISSUE DATE:}

2014-10-07

URL:

http://hdl.handle.net/2433/191123

\section{RIGHT:}

Copyright 2014 American Institute of Physics. This article may be downloaded for personal use only. Any other use requires prior permission of the author and the American Institute of Physics. 


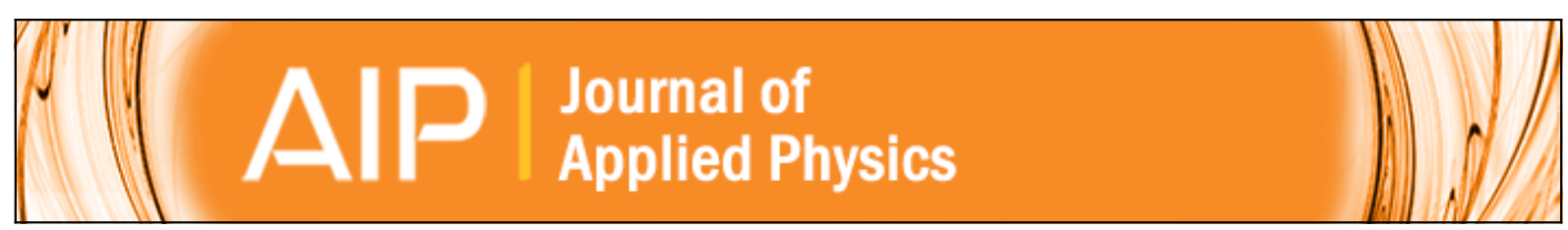

\section{Practical aspects of Kelvin-probe force microscopy at solid/liquid interfaces in various} liquid media

Ken-ichi Umeda, Kei Kobayashi, Noriaki Oyabu, Yoshiki Hirata, Kazumi Matsushige, and Hirofumi Yamada

Citation: Journal of Applied Physics 116, 134307 (2014); doi: 10.1063/1.4896881

View online: http://dx.doi.org/10.1063/1.4896881

View Table of Contents: http://scitation.aip.org/content/aip/journal/jap/116/13?ver=pdfcov

Published by the AIP Publishing

\section{Articles you may be interested in}

Switching spectroscopic measurement of surface potentials on ferroelectric surfaces via an open-loop Kelvin probe force microscopy method

Appl. Phys. Lett. 101, 242906 (2012); 10.1063/1.4772511

High potential sensitivity in heterodyne amplitude-modulation Kelvin probe force microscopy

Appl. Phys. Lett. 100, 223104 (2012); 10.1063/1.4723697

Half-harmonic Kelvin probe force microscopy with transfer function correction

Appl. Phys. Lett. 100, 063118 (2012); 10.1063/1.3684274

Surface potential measurement of organic thin film on metal electrodes by dynamic force microscopy using a piezoelectric cantilever

J. Appl. Phys. 109, 114306 (2011); 10.1063/1.3585865

Practical aspects of Kelvin probe force microscopy

Rev. Sci. Instrum. 70, 1756 (1999); 10.1063/1.1149664

\section{AIP $\mid$ chaos CALL FOR APPLICANTS Seeking new Editor-in-Chief}




\title{
Practical aspects of Kelvin-probe force microscopy at solid/liquid interfaces in various liquid media
}

\author{
Ken-ichi Umeda, ${ }^{1}$ Kei Kobayashi,,${ }^{1,2}$ Noriaki Oyabu,,${ }^{1,3}$ Yoshiki Hirata, ${ }^{4}$ Kazumi Matsushige,,${ }^{1}$ \\ and Hirofumi Yamada ${ }^{1, a)}$ \\ ${ }^{1}$ Department of Electronic Science and Engineering, Kyoto University, Katsura, Nishikyo, Kyoto 615-8510, \\ Japan \\ ${ }^{2}$ The Hakubi Center for Advanced Research, Kyoto University, Katsura, Nishikyo, Kyoto 615-8520, Japan \\ ${ }^{3}$ JST Development of Systems and Technology for Advanced Measurement and Analysis, Honcho, \\ Kawaguchi 332-0012, Japan \\ ${ }^{4}$ Institute for Biological Resources and Functions, National Institute of Advanced Industrial Science and \\ Technology, 1-1-1 Higashi, Tsukuba 305-8566, Japan
}

(Received 30 June 2014; accepted 18 September 2014; published online 3 October 2014)

\begin{abstract}
The distributions of surface charges or surface potentials on biological molecules and electrodes are directly related to various biological functions and ionic adsorptions, respectively. Electrostatic force microscopy and Kelvin-probe force microscopy (KFM) are useful scanning probe techniques that can map local surface charges and potentials. Here, we report the measurement and analysis of the electrostatic and capacitive forces on the cantilever tip induced by application of an alternating voltage in order to discuss the feasibility of measuring the surface charge or potential distribution at solid/liquid interfaces in various liquid media. The results presented here suggest that a nanometer-scale surface charge or potential measurement by the conventional voltage modulation techniques is only possible under ambient conditions and in a non-polar medium and is difficult in an aqueous solution. Practically, the electrostatic force versus dc voltage curve in water does not include the minimum, which is used for the surface potential compensation. This is because the cantilever oscillation induced by the electrostatic force acting on the tip apex is overwhelmed by the parasitic oscillation induced by the electrostatic force acting on the entire cantilever as well as the surface stress effect. We both experimentally and theoretically discuss the factors which cause difficulties in application of the voltage modulation techniques in the aqueous solutions and present some criteria for local surface charge and potential measurements by circumventing these problems. C 2014 AIP Publishing LLC. [http://dx.doi.org/10.1063/1.4896881]
\end{abstract}

\section{INTRODUCTION}

Electrostatic force microscopy $(\mathrm{EFM})^{1,2}$ and Kelvinprobe force microscopy $(\mathrm{KFM})^{3,4}$ are scanning probe techniques based on atomic force microscopy (AFM) that are capable of mapping local surface charge distributions and local surface potentials, respectively, with a high spatial resolution. These methods utilize the detection of the electrostatic forces induced by an alternating voltage applied between the tip and sample surface, and they have been commonly used either in a vacuum environment, under ambient conditions or under non-polar liquid conditions. ${ }^{5}$ Recently, there has been a strong demand for local surface charge and potential measurements in polar liquids, especially in aqueous solutions containing electrolytes, to investigate "in vivo" biological processes, ${ }^{6-8}$ graphene-based electrochemical capacitors, ${ }^{9,10}$ ion-adsorption onto different phases of inorganic minerals, ${ }^{11,12}$ the redox reaction dynamics of molecules, ${ }^{13,14}$ and interfacial chemistry. ${ }^{15}$ However, since the surface charges are screened by the surrounding counter ions in aqueous solutions, forming an electric double layer (EDL), the electrostatic interaction between the tip and surface is not as simple as that in a vacuum or air. Until now,

${ }^{\text {a)} E-m a i l: ~ h-y a m a d a @ k u e e . k y o t o-u . a c . j p ~}$ several researchers have reported the measurements of the EDL force, which is caused by the overlap between the EDLs of two surfaces, as a function of the surface separation to study the local surface charge distribution. ${ }^{16-20}$ Within the past few years, many researchers have investigated the electrostatic force acting on the AFM cantilever with a tip when an ac voltage is applied between the cantilever tip and sample in liquid media., ${ }^{91-40}$ Among these studies, Fukuma et al. proposed a method to estimate the local surface potential in aqueous solutions by the measurements of the first and second harmonic components $\left(1 \omega_{\mathrm{m}}\right.$ and $\left.2 \omega_{\mathrm{m}}\right)$. $^{29}$ Although this method can be directly applied under ambient conditions, for the aqueous solutions, the specific phenomena related to the EDLs should be considered, such as the parasitic cantilever oscillation and the significant reduction of the electrostatic force caused by the EDLs. Very recently, Collins et al. proposed another method to measure the first and second harmonic components based on the time-domain analysis of the cantilever responses instead of the frequency domain. ${ }^{40}$ Up to now, however, there has been no systematic study regarding this topic. We recently published a paper on the possibility of local dielectric property measurements in liquid media and found that the EDL significantly deteriorates the spatial resolution in the local capacitive force measurement. ${ }^{38}$ Here, we discuss the possibility of the local 
surface charge and potential distribution measurements in liquid media using the voltage modulation techniques in detail to present some criteria for achieving the nano-scale surface charge and potential measurements.

We report the measurement and analysis of the electrostatic force on the cantilever with a tip induced by an ac voltage application in various liquid media. We measured the dependence of the electrostatic and capacitive forces on the modulation frequency, externally applied dc voltage, and tip-sample distance. The experimental results are compared with the theoretical models, and the feasibility of measuring the charge distribution or potential at solid/liquid interfaces by the voltage modulation techniques in aqueous solutions is discussed.

The paper is organized as follows. After a brief description of the experimental conditions in Sec. II, we first demonstrate the surface potential measurement in a non-polar liquid medium using KFM in Sec. III. The feasibility of the surface potential measurement in water by the voltage modulation techniques is then discussed in Sec. IV. We calculate the magnitude of the electrostatic force induced by the modulation voltage in aqueous solutions as a function of the modulation frequency and tip-sample distance based on the Poisson-Boltzmann equation in Sec. V. Moreover, we discuss the criteria for the local surface potential measurement in aqueous solutions by comparing the experimental result using a gold-coated colloidal probe and the theoretical model in Sec. VI. Finally, the conclusions are stated in Sec. VII.

\section{MATERIAL AND METHODS}

\section{A. Instruments}

We used a customized AFM instrument (Shimadzu: SPM-9600) with a home-built digital controller programmed using LabVIEW (National Instruments). We used a rectangular cantilever with platinum-iridium coatings on both sides (Nanosensors: PPP-NCSTPt), whose spring constant $\left(k_{z}\right)$ was $5.3 \mathrm{~N} / \mathrm{m}$, calibrated using Sader's method in air. ${ }^{41}$ For the measurement of the dependence of the electrostatic force on the externally applied dc voltage in water, we used a rectangular cantilever with gold coatings on both sides (Nanosensors: PPP-NCHAu), whose nominal spring constant was $42 \mathrm{~N} / \mathrm{m}$, to avoid the variation in the tip-sample distance due to the static deflection caused by the surface stress change. The dimensions of the cantilever were taken from the nominal values. The width $(w)$ and length $(l)$ of the cantilever were 30 and $125 \mu \mathrm{m}$, respectively, and the tip height (h) was $11 \mu \mathrm{m}$. For the experiment described in Sec. VI, we used a colloidal probe (CP) cantilever (sQube: CP-NCH$\mathrm{SiO}-\mathrm{C})$ with gold coatings on both sides, whose nominal spring constant was $42 \mathrm{~N} / \mathrm{m}$. The radius of curvature of the tip $\left(R_{\text {tip }}\right)$ was $3.31 \mu \mathrm{m}$. The nominal values of the dimensions of the CP cantilever are the same as that of PPP-NCHAu. The sensitivity of the deflection sensor was calibrated by the measurement of the thermal noise spectrum before each experiment. A polycrystalline platinum plate was used as a sample except for the experiment in Sec. VI, in which a freshly cleaved graphite was used. A lock-in amplifier with a signal source (AMETEK: 7280) was used to apply an ac voltage and to detect the amplitude and phase of the first or second harmonic components in the cantilever deflection signal.

\section{B. Liquid media}

We performed the experiments in various liquid media such as in air, a fluorocarbon liquid (3M: Fluorinert FC-70) as a non-polar liquid medium, and ultrapure water (Millipore) as an aqueous solution. Reagents were used as supplied by the manufacturers without further purification. The physical properties of the liquid media are shown in Ref. 38 .

\section{KELVIN-PROBE FORCE MICROSCOPY IN NON-POLAR LIQUID MEDIUM}

The electrostatic force acting on the tip and sample in EFM or KFM $\left(F_{\text {esf }}\right)$ is generally described as

$$
F_{\mathrm{esf}}=\frac{1}{2} \frac{\partial C_{\mathrm{ts}}}{\partial z} V^{2}
$$

where $C_{\mathrm{ts}}$ is a capacitance between the tip and sample, $V$ is a bias voltage, and $z$ is the tip-sample distance. When a modulation voltage of an amplitude $V_{\mathrm{ac}}$ at the angular frequency $\omega_{\mathrm{m}}$ with a dc offset voltage $V_{\mathrm{dc}}$, given by $V_{\mathrm{mod}}=V_{\mathrm{dc}}+V_{\mathrm{ac}}$ $\cos \omega_{\mathrm{m}} \mathrm{t}$, is applied between the tip and sample, the electrostatic force becomes

$$
\begin{aligned}
F_{\mathrm{esf}}= & \frac{1}{2} \frac{\partial C_{\mathrm{ts}}}{\partial z}\left[\left(V_{\mathrm{dc}}+V_{\mathrm{SP}}\right)^{2}+\frac{1}{2} V_{\mathrm{ac}}^{2}\right. \\
& \left.+2\left(V_{\mathrm{dc}}+V_{\mathrm{SP}}\right) V_{\mathrm{ac}} \cos \omega_{\mathrm{m}} t+\frac{1}{2} V_{\mathrm{ac}}^{2} \cos 2 \omega_{\mathrm{m}} t\right]
\end{aligned}
$$

where $V_{\mathrm{SP}}$ is the surface potential difference between the tip and sample. The third and fourth terms are the $1 \omega_{\mathrm{m}}$ and $2 \omega_{\mathrm{m}}$ components, respectively. The electrostatic force of the $2 \omega_{\mathrm{m}}$ component is often referred to as the capacitive force. In KFM, $V_{\mathrm{SP}}$ can be measured by controlling $V_{\mathrm{dc}}$ to null the $1 \omega_{\mathrm{m}}$ component.

We first conducted the KFM measurements on a pn-patterned silicon sample ${ }^{42}$ in air and a non-polar liquid medium, a fluorocarbon liquid. In these experiments, the tip-sample distance was regulated by the intermittent-contact mode, and the modulation frequency, $f_{\mathrm{m}}\left(=\omega_{\mathrm{m}} / 2 \pi\right)$ was set at $2 \mathrm{kHz}$. Figures 1(a) and 1(b) show topographic images of the pnpatterned silicon sample in air and fluorocarbon liquid, respectively. Higher square areas with a side of $5 \mu \mathrm{m}$ are $p$ and $n$-type regions, while the lower background area is the highly doped- $n$-type $\left(n^{+}\right.$-type) region. Figures $1(\mathrm{c})$ and $1(\mathrm{~d})$ show surface potential images, which were simultaneously obtained with the topographic images in air and fluorocarbon liquid, respectively. The surface potentials of the $p$ - and $n$ type regions were found to be higher than the $n^{+}$-type region independent of the measurement environments. The result demonstrates that the local potential measurements can be performed in air and non-polar liquid media without any problems, as also reported in Ref. 5. 
(a) Topography in Air

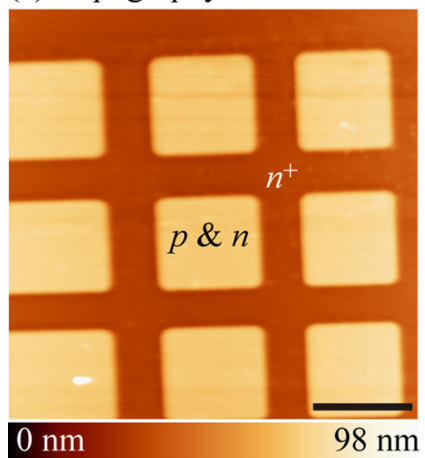

(b) Topography in FC-70

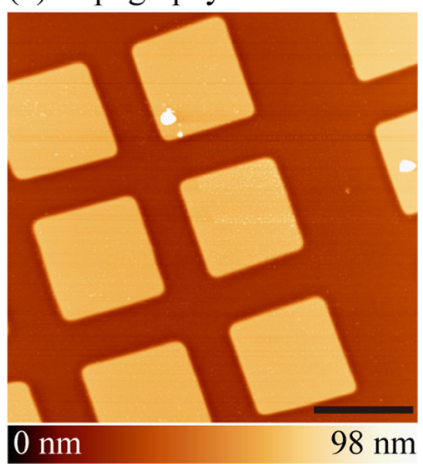

(c) SP in Air

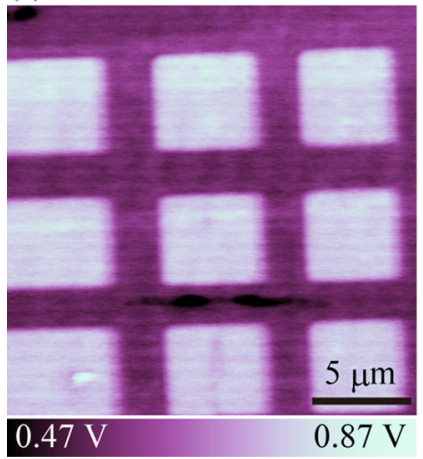

(d) SP in FC-70

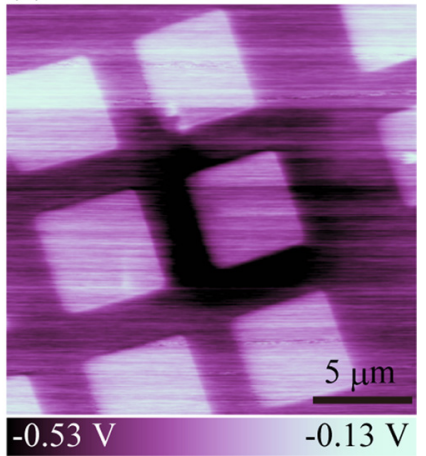

FIG. 1. Topographic images of pn-patterned silicon sample obtained in (a) air and (b) fluorocarbon liquid. Surface potential (SP) images simultaneously observed in (c) air and (d) fluorocarbon liquid.

\section{ELECTROSTATIC FORCE MEASUREMENTS IN AQUEOUS SOLUTION}

Here, we discuss the feasibility of the local surface potential measurement in water by the voltage modulation techniques. We start with the choice of the right modulation frequency range. Figure 2(a) shows a plot of the amplitude of the $1 \omega_{\mathrm{m}}$ component in the cantilever deflection signal measured in air as a function of $f_{\mathrm{m}}$ when $V_{\mathrm{dc}}$ and $V_{\mathrm{ac}}$ were set at $6 \mathrm{~V}$ and $2.8 \mathrm{~V}$ peak-to-peak, respectively. The distance between the cantilever and sample was about $10 \mu \mathrm{m}$. The solid curve is an experimental result and the broken curve is a theoretical fit to the experimental curve. The first harmonic complex amplitude of the cantilever induced by the electrostatic force $\left(A_{\text {esf }}^{1 \omega_{\mathrm{m}}}\right)$ was calculated as

$$
A_{\mathrm{esf}}^{1 \omega_{\mathrm{m}}}=G_{\mathrm{cl}} F_{\mathrm{esf}}^{1 \omega_{\mathrm{m}}}=\frac{Q}{Q\left[1-\left(f_{\mathrm{m}} / f_{1}\right)^{2}\right]+\mathrm{i}\left(f_{\mathrm{m}} / f_{1}\right)} \frac{1}{k_{z}} F_{\mathrm{esf}}^{1 \omega_{\mathrm{m}}},
$$

where $G_{\mathrm{cl}}$ and $F_{\mathrm{esf}}^{1 \omega_{\mathrm{m}}}$ are the transfer function of the cantilever as a damped oscillator model and the first harmonic electrostatic force, respectively. $f_{1}$ is the first flexural resonance frequency and $Q$ is the quality factor of the cantilever (at $f_{1}$ in this case). On the other hand, Fig. 2(b) shows a plot of the amplitude of the $1 \omega_{\mathrm{m}}$ component in the cantilever deflection signal as a function of $f_{\mathrm{m}}$ in water, when $V_{\mathrm{dc}}$ and $V_{\mathrm{ac}}$ were set at $0 \mathrm{~V}$ and $2.8 \mathrm{~V}$ peak-to-peak, respectively. The distance between the tip apex and sample was about $10 \mu \mathrm{m}$. As we previously reported in Ref. 28, the cantilever deflection was induced by the surface stress, ${ }^{43}$ especially in the low

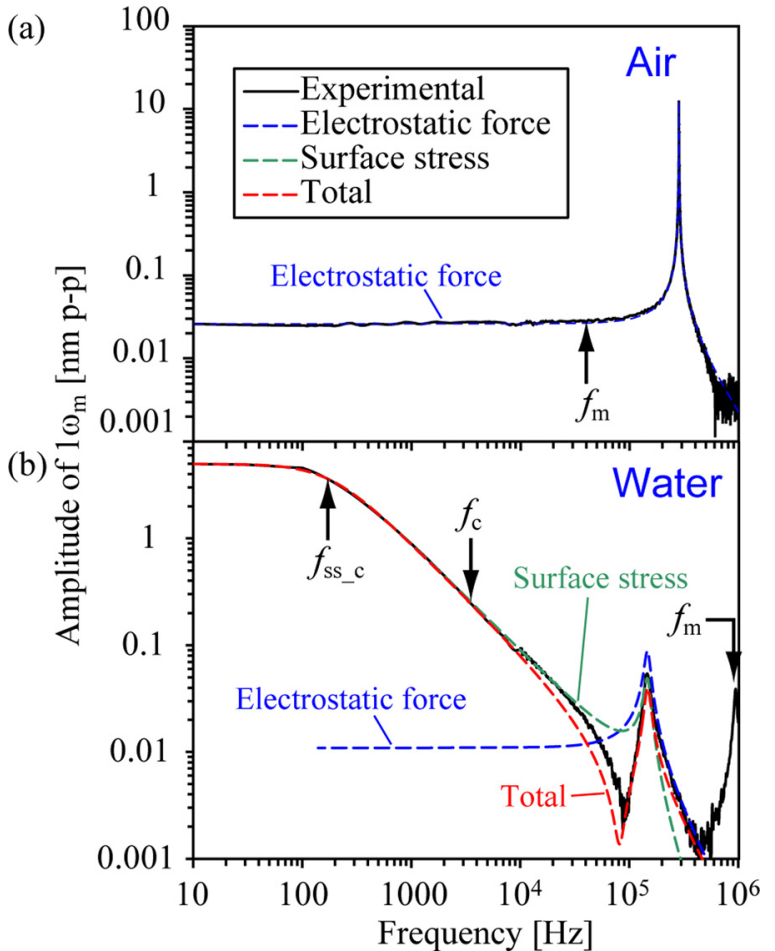

FIG. 2. Plots of amplitude of $1 \omega_{\mathrm{m}}$ component in cantilever deflection signal as a function of $f_{\mathrm{m}}$ in (a) air and (b) water. Broken curve in (a) is a fitted curve using a damped harmonic oscillator model. Broken curves in (b) are fitted curves for surface stress contribution (green) and electrostatic force contribution (blue), and their sum (red).

frequency range, as well as by the electrostatic force in the high frequency range. A dip at the frequency of around 100 $\mathrm{kHz}$ was caused by the antiphase between the surface stress and the electrostatic force contributions. This frequency is referred to as the transition frequency $\left(f_{t}\right)$ of the contributions hereafter. Here, the first harmonic complex amplitude $\left(A^{1 \omega_{\mathrm{m}}}\right)$ is modeled as the sum of $F_{\text {esf }}^{1 \omega_{\mathrm{m}}}$ and surface stress $F_{\mathrm{ss}}^{1 \omega_{\mathrm{m}}}$ as expressed by

$$
\begin{aligned}
A^{1 \omega_{\mathrm{m}}} & =G_{\mathrm{cl}}\left(F_{\mathrm{ss}}^{1 \omega_{\mathrm{m}}}+F_{\mathrm{esf}}^{1 \omega_{\mathrm{m}}}\right) \\
& =G_{\mathrm{cl}}\left[\frac{1}{1+\mathrm{i}\left(f / f_{\mathrm{ss} \_\mathrm{c}}\right)^{\alpha}} F_{\mathrm{ss} 0}+F_{\mathrm{esf}}^{1 \omega_{\mathrm{m}}}\right],
\end{aligned}
$$

where $F_{\text {ss } 0}$ and $f_{\text {ss_c }}$ are fitting parameters, i.e., a complex effective surface stress force $\left(\left|F_{\mathrm{ss} 0}\right| \mathrm{e}^{-\mathrm{i} \theta_{\mathrm{ss} 0}}\right)$ at a low-frequency limit and a cutoff frequency, respectively. ${ }^{28}$ The two broken curves in Fig. 2(b) are the fitted curves for the surface stress contribution (green) and the electrostatic force contribution (blue). $F_{\text {esf }}, F_{\text {ss } 0}, \theta_{\text {ss } 0}$, and $f_{\text {ss_c }}$ determined by the fitting were $0.31 \mathrm{nN}$ peak-to-peak, $140 \mathrm{nN}$ peak-to-peak, $-85^{\circ}$, and $180 \mathrm{~Hz}$, respectively, and $\alpha$ was almost 1 . The surface potential of the cantilever $\left(\psi_{0}\right)$ estimated from the value of $F_{\mathrm{ss} 0}$ was about $0.57 \mathrm{~V}$ (see Appendix A). Since the deflection induced by the surface stress variation does not contain any information on the local charge or potential of the sample surface, we focused on the electrostatic force contribution hereafter. As the tip-sample distance in this experiment was much larger than the Debye length, the electrostatic force observed in the high frequency range was not caused by the 
overlap of the EDLs of the tip and sample, and it did not also contain any information on the local charge distribution or surface potential. For the local surface charge or potential measurement, the electrostatic force should exhibit a dependence on the tip-sample distance when the tip-sample distance is reduced to the same order of magnitude as the Debye length.

In order to know whether or not the KFM feedback can be used, we investigated the dependence of the amplitude of the harmonic components in the deflection signal on $V_{\mathrm{dc}}$ and the tip-sample distance. We fixed $f_{\mathrm{m}}$ at the second resonance frequency of the cantilever $\left(f_{2}=933 \mathrm{kHz}\right)$, which is in the frequency range where the electrostatic force contribution becomes dominant. Figures 3(a) and 3(b) show plots of the amplitude of the $1 \omega_{\mathrm{m}}$ and $2 \omega_{\mathrm{m}}$ components as a function of $V_{\mathrm{dc}}$, obtained when the tip-sample distance was kept at about $5 \mathrm{~nm}$, respectively. The measurements were conducted in air and pure water. In air, the amplitude of the $1 \omega_{\mathrm{m}}$ component became zero when the condition $V_{\mathrm{dc}}=-V_{\mathrm{SP}}$ is met (see Eq. (2)), and the plot showed a symmetric V-shape as expected. However, as shown in Fig. 3(a), in pure water, the amplitude of the $1 \omega_{\mathrm{m}}$ component did not show the V-shape, and the forward and backward traces were different, indicating a poor reproducibility of the surface conditions. On the other hand, plots of the amplitude of the $2 \omega_{\mathrm{m}}$ component in Fig. 3(b) showed almost no dependence on $V_{\mathrm{dc}}$, both in air and in water with a good reproducibility, as expected from Eq. (2). Figures 3(c) and 3(d) show plots of the amplitudes of the $1 \omega_{\mathrm{m}}$ and $2 \omega_{\mathrm{m}}$ components as a function of the tipsample distance, respectively. The origin of the tip-sample distance was determined at the position at which the amplitudes of both components began to drop because of the tip contact. In air, as the tip-sample distance was decreased, the magnitude of both components became larger because of the increase in the tip-sample capacitance. However, in water, the $2 \omega_{\mathrm{m}}$ component showed almost no increase, and the $1 \omega_{\mathrm{m}}$ component even decreased with the decrease in the tip-sample distance. In Sec. V, we theoretically model the electrostatic force acting between a cantilever and a tip in aqueous solutions to discuss the origin of these discrepancies.

\section{THEORETICAL MODELS OF ELECTROSTATIC FORCE IN AQUEOUS SOLUTION}

In this section, we discuss the theoretical models of the electrostatic force in water in order to explain the experimental results. As shown in Eq. (2), the $1 \omega_{\mathrm{m}}$ component of the electrostatic force is proportional to a product of the magnitude of the ac modulation voltage and the difference in the electrostatic potential difference. First, we discuss the equivalent circuit of aqueous solution between the cantilever and sample surface to derive the criteria for the modulation frequency at which the ac modulation voltage is effectively applied between the tip and sample surface. Second, we calculate the electrostatic potential profile between the tip and sample surface to estimate the electrostatic force acting on the tip in aqueous solutions.

\section{A. Criteria for modulation frequency}

The electrode/electrolyte interface is often described by the Gouy-Chapman-Stern (GCS) model, in which the EDL is composed of two layers; i.e., the Stern layer and the diffuse layer. ${ }^{44}$ Although the charge dynamics at the interface is complicated and difficult to be represented as a simple equivalent circuit, the Randles circuit ${ }^{45}$ has been widely accepted in the field of electrochemistry ${ }^{44}$ and impedance spectroscopy. ${ }^{46}$ This equivalent circuit is valid only when the EDLs of the surfaces do not overlap with each other. Figure 4(a) shows a general Randles circuit, referred to as (A) hereafter, in which the EDL capacitance $C_{\mathrm{EDL}}$, comprised of a series of the Stern layer capacitance $C_{\mathrm{S}}$ and the diffuse layer capacitance $C_{\mathrm{D}}$, is connected in parallel with a series of the charge transfer resistance $R_{\mathrm{CT}}$ and the Warburg impedance $Z_{\mathrm{W}}{ }^{44}$ (a)

(c)
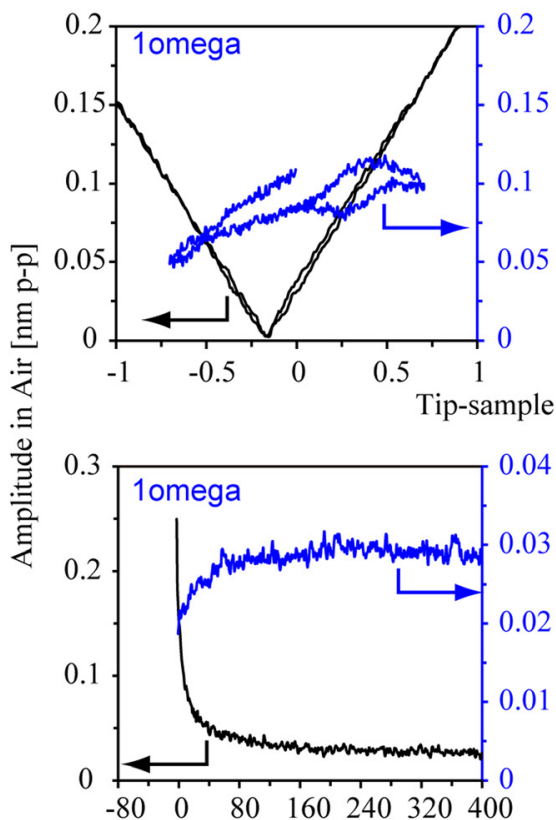

(b)

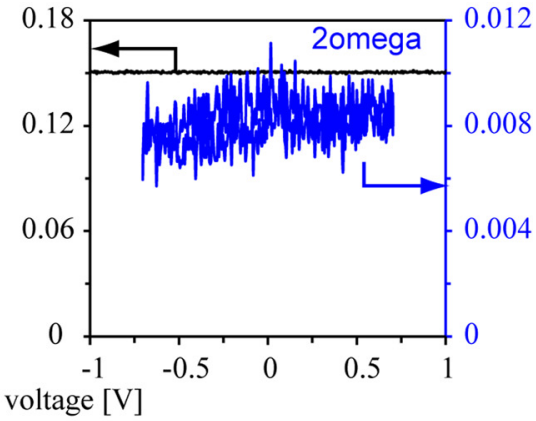

(d)

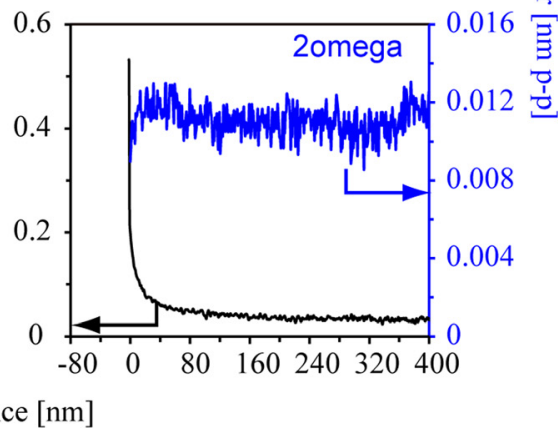

FIG. 3. Plots of amplitude of (a) $1 \omega_{\mathrm{m}}$ and (b) $2 \omega_{\mathrm{m}}$ component as a function of $V_{\mathrm{dc}}$ in air and water. Plots of amplitudes of (c) $1 \omega_{\mathrm{m}}$ and (d) $2 \omega_{\mathrm{m}}$ components in cantilever deflection signal as a function of the tip-sample distance in air $\left(f_{\mathrm{m}}=30 \mathrm{kHz}\right)$ and water $\left(f_{\mathrm{m}}=f_{2}=933 \mathrm{kHz}\right)$.

Tip-sample distance [nm] 


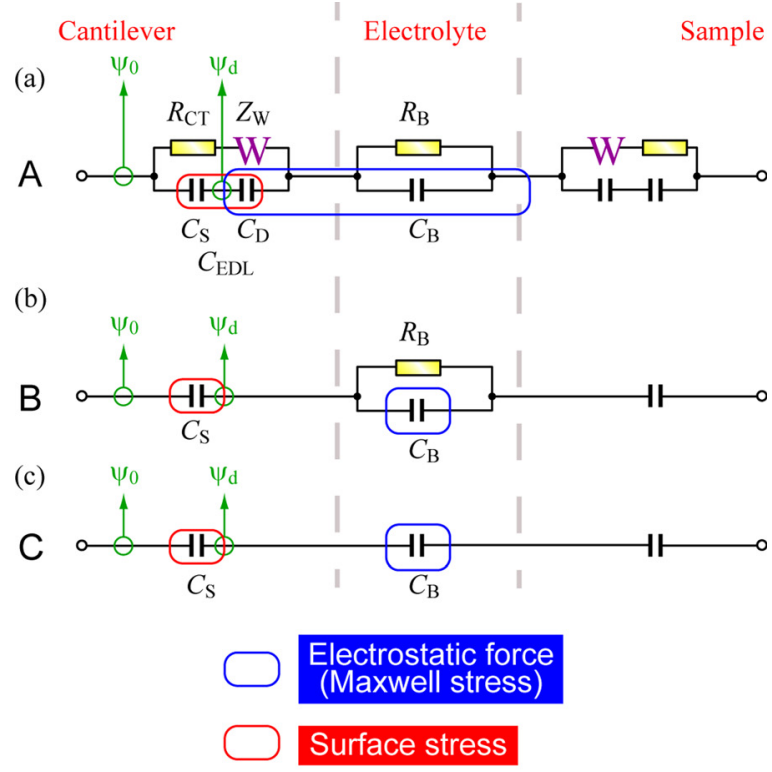

(d)

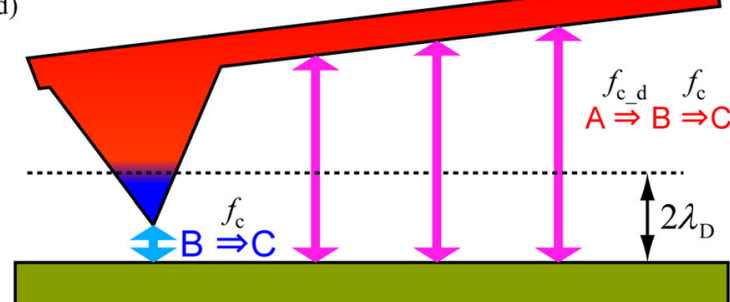

FIG. 4. Equivalent circuit of aqueous solution between a cantilever and a sample surface based on Randles circuit (a), and actual equivalent circuit for (b) at a modulation frequency with the range $f_{\mathrm{c}_{-} \mathrm{d}}<f_{\mathrm{m}}<f_{\mathrm{c}}$, and (c) at a very high modulation frequency $\left(f_{\mathrm{m}}>f_{\mathrm{c}}, f_{\mathrm{c} \_\mathrm{d}}\right)$. (d) Schematic of transition of equivalent circuits depending on the part of the cantilever.

The impedance of the bulk liquid is expressed by a parallel circuit of the bulk solution resistance $R_{\mathrm{B}}$ and capacitance $C_{\mathrm{B}}$.

The Poisson-Boltzmann (P-B) equation for a two parallel flat plate system in a 1-1 symmetric electrolyte is given by

$$
\frac{\mathrm{d}^{2} \psi}{\mathrm{d} x^{2}}=\frac{e n_{\infty}}{\varepsilon_{0} \varepsilon_{\mathrm{r}}} \sinh \left(\frac{e \psi}{k_{\mathrm{B}} T}\right)
$$

where $\psi$ represents the electrostatic potential. When an ac voltage is applied to the electrode/electrolyte interface, charge, and discharge of the diffuse layer occur with an ionic current flow in the bulk solution. Solving Eq. (5) gives $C_{\mathrm{D}}$ as

$$
C_{\mathrm{D}}=\kappa_{\mathrm{D}} \varepsilon_{0} \varepsilon_{\mathrm{r}} \cosh \left(\frac{e V_{\mathrm{ac} \_\mathrm{d}}}{2 k_{\mathrm{B}} T}\right)
$$

where $\kappa_{\mathrm{D}}, \varepsilon_{0}, \varepsilon_{\mathrm{r}}, e, V_{\mathrm{ac} \mathrm{d}}, k_{\mathrm{B}}$, and $T$ are the inverse of the Debye length $\left(\lambda_{\mathrm{D}}\right)$, the dielectric constant of a vacuum, relative dielectric constant, the elementary charge, ac voltage drop in $C_{\mathrm{D}}$, and the Boltzmann constant and temperature, respectively. ${ }^{44} \kappa_{\mathrm{D}}$ is given by

$$
\kappa_{\mathrm{D}}=\lambda_{\mathrm{D}}^{-1}=\left(\frac{2 e^{2} n_{\infty}}{\varepsilon_{0} \varepsilon_{\mathrm{r}} k_{\mathrm{B}} T}\right)^{1 / 2} .
$$

The cantilever deflection caused by the surface stress variation is prominent when most of the voltage is applied to
$C_{\mathrm{D}}$, while the contribution of the electrostatic force becomes dominant when most of the voltage is applied to $C_{\mathrm{B}} \cdot{ }^{47,48} \mathrm{~A}$ threshold frequency $\left(f_{\mathrm{c} \_\mathrm{d}}\right)$ is defined as the frequency at which the impedance of $C_{\mathrm{D}}$ becomes smaller than the bulk impedance $\left(R_{\mathrm{B}}\right.$ and $\left.C_{\mathrm{B}}\right)$, i.e., $f_{\mathrm{c} \_\mathrm{d}}=1 /\left[2 \pi R_{\mathrm{B}}\left(C_{\mathrm{D}} / 2\right)\right]$. When $f_{\mathrm{m}}$ is higher than $f_{\mathrm{c} \_\mathrm{d}}$, the applied voltage becomes effectively applied to $C_{\mathrm{B}}$ and $R_{\mathrm{B}}$. In this case, a simplified Randles circuit (B) can be used as shown in Fig. 4(b). As $R_{\mathrm{B}}$ is described as

$$
R_{\mathrm{B}}=d \rho_{\mathrm{B}},
$$

where $d$ and $\rho_{\mathrm{B}}$ are the distance between the sample and each part of the cantilever and the resistivity of the electrolyte, respectively, $f_{\mathrm{c} \_\mathrm{d}}$ is given by

$$
f_{\mathrm{c} \_\mathrm{d}}\left(d, V_{\mathrm{ac} \_\mathrm{d}}\right)=\frac{1}{2 \pi R_{\mathrm{B}}\left(C_{\mathrm{D}} / 2\right)}=\frac{1}{\pi d \kappa_{\mathrm{D}} \varepsilon_{0} \varepsilon_{\mathrm{r}} \rho_{\mathrm{B}} \cosh \left(\frac{e V_{\mathrm{ac} \_\mathrm{d}}}{2 k_{\mathrm{B}} T}\right)} .
$$

Note that $C_{\mathrm{S}}$ and $R_{\mathrm{B}}$ are functions of $V_{\mathrm{ac} \text { d }}$ and $d$, which are not unique, but different for individual lines of electric flux between the tip and sample, therefore $f_{\text {c_d }}$ is also different for each part of the cantilever. When the tip apex is outside the EDL of the sample surface, only the cantilever part should be taken into account. The cantilever deflection caused by the surface stress variation is prominent when most of the voltage is applied to $C_{\mathrm{D}}$, while the contribution of the electrostatic force becomes dominant when most of the voltage is applied to $C_{\mathrm{B}} \cdot{ }^{47,48}$ Therefore, $f_{\mathrm{c} \_\mathrm{d}}$ is equivalent to $f_{\mathrm{ss} \_\mathrm{c}}$. The nearer the cantilever is located to the sample surface, the higher $f_{\text {ss_c }}$.

When $f_{\mathrm{m}}$ is even higher than another threshold frequency $\left(f_{\mathrm{c}}\right)$, the impedance of $C_{\mathrm{B}}$ becomes smaller than $R_{\mathrm{B}}$. In this case, a further simplified equivalent circuit $(C)$ can be used, as shown in Fig. 4(c). $f_{\mathrm{c}}$ is the characteristic relaxation frequency of the ionic current flow, which is dependent only on the physical property of a solvent, given by

$$
f_{\mathrm{c}}=\frac{1}{2 \pi \varepsilon_{0} \varepsilon_{\mathrm{r}} \rho_{\mathrm{B}}} \propto \frac{n_{\infty}}{\varepsilon_{\mathrm{r}}},
$$

where $n_{\infty}$ is the number density of ions in the bulk solution. In the case of pure water with dissolved $\mathrm{CO}_{2}$ gas, $f_{\mathrm{c}}$ is around $10 \mathrm{kHz}$. Note that $f_{\mathrm{c} \_\mathrm{d}}$ is expressed using $f_{\mathrm{c}}$ as

$$
f_{\mathrm{c} \_\mathrm{d}}\left(d, V_{\text {ac_d }}\right)=\frac{2 f_{\mathrm{c}}}{d \kappa_{\mathrm{D}} \cosh \left(\frac{e V_{\mathrm{ac} \_\mathrm{d}}}{2 k_{\mathrm{B}} T}\right)} \propto n_{\infty}^{1 / 2} .
$$

When the tip is brought closer to the sample surface than twice the length of $\lambda_{\mathrm{D}}\left(z<2 \lambda_{\mathrm{D}}\right)$, the EDLs begin to overlap with each other. In this case, $f_{\text {c_d }}$ for the tip part is expressed as

$$
f_{\mathrm{c}_{-} \mathrm{d}}\left(2 \kappa_{\mathrm{D}}^{-1}, V_{\mathrm{ac} \_\mathrm{d}}\right)=\frac{f_{\mathrm{c}}}{\cosh \left(\frac{e V_{\mathrm{ac} \_\mathrm{d}}}{2 k_{\mathrm{B}} T}\right)} .
$$

Since $C_{\mathrm{D}}$ which is dependent on $V_{\text {ac_d }}, V_{\text {ac_d }}$ can be calculated by solving the following equation: 


$$
V_{\mathrm{ac} \_\mathrm{d}}=\frac{V_{\mathrm{ac} \_\mathrm{d}} / 2}{1+\frac{\delta C_{\mathrm{D}}}{\delta C_{\mathrm{S}}}}=\frac{V_{\mathrm{ac} \_\mathrm{d}} / 2}{1+\varepsilon_{0} \varepsilon_{\mathrm{r}} \kappa_{\mathrm{D}} \cosh \left(\frac{e V_{\mathrm{ac} \_\mathrm{d}}}{2 k_{\mathrm{B}} T}\right) / \delta C_{\mathrm{S}}},
$$

where $\delta C_{\mathrm{D}}$ and $\delta C_{\mathrm{S}}$ are the diffuse layer capacitance and the Stern layer capacitance per unit area on the tip, respectively. In the case when $V_{\text {ac_d }}$ is more than hundreds of $m V, f_{\mathrm{c}_{-} \mathrm{d}}$ in the 1-1 symmetric electrolyte solution becomes much lower than $f_{\mathrm{c}}$ with a concentration of less than $0.1 \mathrm{mM}$, while $f_{\mathrm{c} \_\mathrm{d}}$ is almost the same as $f_{\mathrm{c}}$ with a concentration of more than $10 \mathrm{mM}$.

Figure 4(d) summarizes the equivalent circuits depending on part of the cantilever. The cantilever and tip parts far from the sample surface $\left(z>2 \lambda_{\mathrm{D}}\right)$ are characterized by the equivalent circuit (A) for the low frequency range, (B) for $f_{\mathrm{c} \_\mathrm{d}}<f_{\mathrm{m}}<f_{\mathrm{c}}$, and then (C) for $f_{\mathrm{m}}>f_{\mathrm{c}}$ (see red region). The tip part closer to the sample surface $\left(z<2 \lambda_{\mathrm{D}}\right)$ is characterized by the equivalent circuit (B) for $f_{\mathrm{m}}<f_{\mathrm{c}}$ and (C) for $f_{\mathrm{m}}>f_{\mathrm{c}}$ (see blue region).

\section{B. Theoretical electric potential profiles and electrostatic forces}

Next, we discuss the electric potential profiles between the tip and sample surface. First, we calculated the electric field induced by the externally applied ac voltage $\left(V_{\mathrm{ac}}\right)$. Based on the equivalent circuit (A), $V_{\text {ac }}$ applied to $C_{\mathrm{D}}\left(V_{\text {ac_d }}\right)$ is used to solve the P-B equation, while the $V_{\mathrm{ac}}$ drop in $C_{\mathrm{B}}$ is used to solve the Laplace equation. Figure 5(a) shows the ac potential profiles between the electrodes with a large distance calculated with the constant potential boundary conditions $^{49}$ as a function of $f_{\mathrm{m}}$. The curves depict the potential profiles when the maximum positive voltage was applied to the left electrode assuming the tip (T) relative to the right electrode assuming the sample (S). The gradient of the profiles in this figure are symmetric, however the potential profiles across the tip and sample might be asymmetric due to their geometries. As $f_{\mathrm{m}}$ increases, $V_{\text {ac_d }}$ decreases and eventually the gradient of the potential (electric field) becomes uniform. Figure 5(b) shows the ac potential profiles between the electrodes with a short distance calculated as a function of $f_{\mathrm{m}}$. As discussed above, in this situation the EDLs overlap with each other, therefore we used the Laplace equation to calculate the potential profiles. The electric field in $C_{\mathrm{B}}$ increases with increasing $f_{\mathrm{m}}$, as shown in the figure. Figure 5(c) shows a comparison of the potential profiles for large and short distances with $f_{\mathrm{m}}$ higher than $f_{\mathrm{c}}$. As the distance decreases, $C_{\mathrm{B}}$ increases, while the potential drop in $C_{\mathrm{S}}$ also increases. Note that $V_{\text {ac }}$ mainly drops in $C_{\mathrm{S}}$, and partially applied to $C_{\mathrm{B}}$, especially in strong electrolytes whose $C_{\mathrm{B}}$ and $R_{\mathrm{B}}$ are quite low.
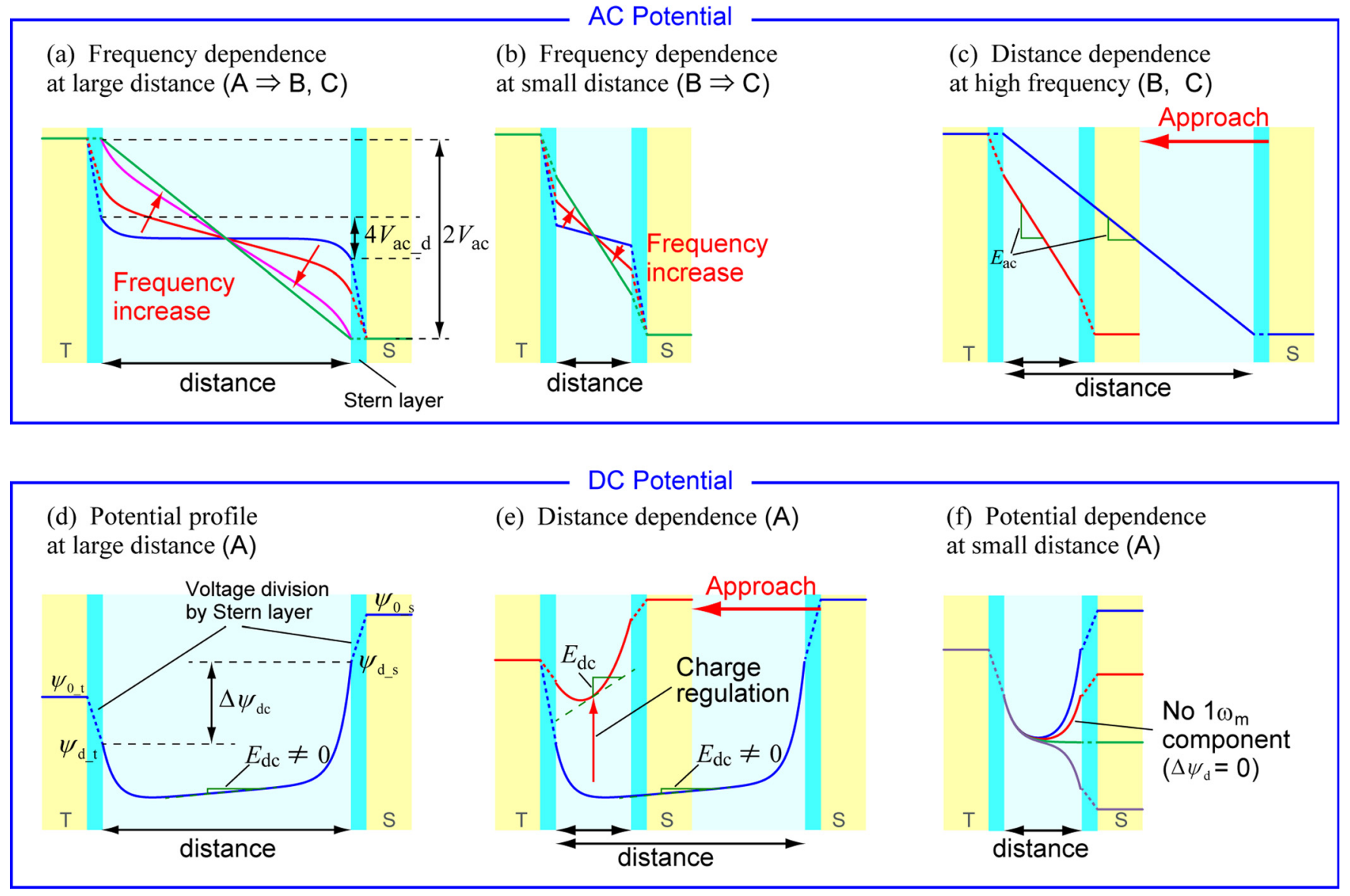

FIG. 5. Schematics of ac potential profiles in aqueous solution as a function of $f_{\mathrm{m}}$ for electrodes with a large distance (a) and with a short distance (b). (c) Schematic of ac potential profiles in solution as a function of tip-sample distance. These ac potential profiles depict the instantaneous maximum voltages in the electrolyte observed when $V_{\text {ac }}$ is applied to the left electrode assuming the tip (T) is higher than the right electrode as the sample (S). Schematics of dc potential profiles for electrodes with a large distance (d) and with a short distance (e). (f) Schematics of dc potential profiles for electrodes with a short distance as a function of $V_{\mathrm{dc}}$ applied to the sample. 
As discussed later, this phenomenon deteriorates the spatial resolution of the electrostatic force as well as the capacitive force measurements. ${ }^{38}$ Note that these potential profiles were calculated based on the equivalent circuits discussed above. The calculation of the potential profiles for the low frequency range $\left(f_{\mathrm{m}}<f_{\mathrm{c} \_\mathrm{d}}\right)$ requires a numerical treatment of the Poisson-Nernst-Planck equation. ${ }^{50}$

Second, we calculated the electric field induced by an externally applied dc voltage $\left(V_{\mathrm{dc}}\right)$. There are two characteristic potentials in aqueous solutions, the absolute electrode potential $\left(\psi_{0}\right)$, and the surface potential at the diffuse layer $\left(\psi_{\mathrm{d}}\right)$. While the potential is always referenced to the potential of the bulk solution, the former refers to the potential of the inner part of $C_{\mathrm{S}}$, and the latter refers to the potential of the outer part of $C_{\mathrm{S}}$ or the inner part of $C_{\mathrm{D}}$. Despite the fact that the theory for the analysis of the dc potential is more established than that for the ac potential, it is difficult to calculate the dc potential profiles including the Stern layers. This is because there is no technique that directly measures electrostatic potentials at arbitrary positions in the electrode/electrolyte interface, especially in the Stern layer. Figure 5(d) shows the calculated dc potential profiles at a large distance.
In the GCS model, the potential gradient should be almost zero at the mid-point between the electrodes with a large separation. However, as we experimentally observed the electrostatic force even when the cantilever was far from the sample surface, we added a slope to the potential profile considering a leakage current in the EDL. Note that the magnitude of the dc electric field is not to scale in the figure; the electric field in the Stern layer is actually much larger than that in the diffuse layer in many cases. As the two surfaces come close to each other, $\psi_{\mathrm{d}}$ of both surfaces vary because of the charge regulation as shown in Fig. 5(e). The boundary conditions for both the ac and dc potential profiles are near the constant potential in the case of metal surfaces, however an unpredicted charge regulation might occur by the electrochemical reaction and desorption/adsorption of ions even in the case of metal surfaces. ${ }^{51,52}$

While the surface stress is induced by $V_{\mathrm{dc}}$ and $V_{\mathrm{ac}}$ applied to $C_{\mathrm{EDL}}$, the electrostatic force is induced by $V_{\mathrm{dc}}$ and $V_{\mathrm{ac}}$ drops in $C_{\mathrm{B}}$. Therefore, the electrostatic force per unit area $\left(T_{\text {esf }}\right)$ can be expressed using the dc electric field $E_{\mathrm{dc}}(x)$ and ac electric field $E_{\mathrm{ac}}\left(x, \omega_{\mathrm{m}}\right)$ at the mid-point $(x=d / 2)$ as

$$
\begin{aligned}
T_{\mathrm{esf}}\left(\omega_{\mathrm{m}}\right) & =\frac{1}{2} \varepsilon_{0} \varepsilon_{\mathrm{r}} E^{2}=\frac{1}{2} \varepsilon_{0} \varepsilon_{\mathrm{r}}\left[E_{\mathrm{dc}}(d / 2)+E_{\mathrm{ac}}\left(d / 2, \omega_{\mathrm{m}}\right) \cos \omega_{\mathrm{m}} t\right]^{2}, \\
& =\frac{1}{2} \varepsilon_{0} \varepsilon_{\mathrm{r}}\left\{\begin{array}{l}
{\left[E_{\mathrm{dc}}(d / 2)\right]^{2}+\frac{1}{2}\left[E_{\mathrm{ac}}\left(d / 2, \omega_{\mathrm{m}}\right)\right]^{2}} \\
+2\left[E_{\mathrm{dc}}(d / 2)\right]\left[E_{\mathrm{ac}}\left(d / 2, \omega_{\mathrm{m}}\right)\right] \cos \omega_{\mathrm{m}} t+\frac{1}{2}\left[E_{\mathrm{ac}}\left(d / 2, \omega_{\mathrm{m}}\right)\right]^{2} \cos 2 \omega_{\mathrm{m}} t
\end{array}\right\} .
\end{aligned}
$$

Furthermore, the dielectric saturation should be taken into account for calculating the electrostatic force, since a high ac electric field applied between the tip and sample causes the significant decrease in the orientation of solvent molecules (discussed later). Figure 5(f) shows dc potential profiles as a function of $V_{\mathrm{dc}}$. When the diffuse potential of the tip and sample become exactly the same $\left(\Delta \psi_{\mathrm{d}}=0\right)$, the electrostatic force is nulled.

Finally, we discuss the origin of the electrostatic force observed in the high frequency range of the spectrum shown in Fig. 2(b). Since the spectrum was obtained when the distance between the tip and the sample is much larger than $\lambda_{\mathrm{D}}$, this electrostatic force cannot be caused by the overlap between the EDLs. If we divide the electrostatic force acting on a cantilever with a tip into three parts, $F_{\text {esf }}^{1 \omega_{\mathrm{m}}}=F_{\text {apex }}^{1 \omega_{\mathrm{m}}}+F_{\text {cone }}^{1 \omega_{\mathrm{m}}}+F_{\mathrm{cl}}^{1 \omega_{\mathrm{m}}}, F_{\mathrm{cl}}^{1 \omega_{\mathrm{m}}}$ is dominant over the other components. By using the equation for $F_{\mathrm{cl}}^{1 \omega_{\mathrm{m}}}$ in Ref. 34 which considers the non-uniform distribution of the electrostatic force ${ }^{53,54}$ with an approximation for the parallel plate system, $F_{\mathrm{esf}}^{1 \omega_{\mathrm{m}}}$ is given by

$$
\begin{aligned}
F_{\mathrm{esf}}^{1 \omega_{\mathrm{m}}} & \approx F_{\mathrm{cl}}^{1 \omega_{\mathrm{m}}}=\varepsilon_{0} \varepsilon_{\mathrm{r}} E_{\mathrm{dc}} E_{\mathrm{ac}} w \int_{0}^{l} \frac{\phi_{1}(l-x)}{\phi_{1}(l)} \mathrm{d} x \\
& \approx \frac{3}{8} l w \varepsilon_{0} \varepsilon_{\mathrm{r}} E_{\mathrm{dc}} E_{\mathrm{ac}},
\end{aligned}
$$

where $E_{\mathrm{dc}}$ is the dc electric field, $\phi_{n}$ is the eigenfunction of the $n$th eigenmode, $r_{0}=\frac{z+h}{2} \cot \left(\frac{\theta_{\mathrm{cl}}}{2}\right)$, and $\theta_{\mathrm{cl}}$ is the tilt angle of the cantilever. $\phi_{n}$ is given by

$$
\begin{aligned}
\phi_{n}(x)= & \left(\cos k_{n} x-\cosh k_{n} x\right)-\frac{\cos k_{n} l+\cosh k_{n} l}{\sin k_{n} l+\sinh k_{n} l} \\
& \times\left(\sin k_{n} x-\sinh k_{n} x\right),
\end{aligned}
$$

where $k_{n} l$ is the $n$th eigenvalue. By fitting Eq. (3) using Eq. (15) to the experimental curve in the high frequency range in Fig. 2(b) (blue dotted line), $E_{\mathrm{dc}}$ was estimated to be $2.5 \times 10^{3} \mathrm{~V} / \mathrm{m}$ and $\Delta \psi_{\mathrm{d}}\left(=E_{\mathrm{dc}} \cdot(z+h)\right)$ was estimated to be $0.05 \mathrm{~V}$. This long-range electrostatic force cannot be explained by the P-B equation. Even if assuming that the surface potential difference is $1 \mathrm{~V}, \Delta \psi_{\mathrm{d}}$ estimated by solving the $\mathrm{P}-\mathrm{B}$ equation is on the order of only $10^{-21} \mathrm{~V} . E_{\mathrm{dc}}$ is also estimated to be as small as $10^{-16} \mathrm{~V} / \mathrm{m}$, which is much less than the value calculated from the experiment. In pure water or such high impedance solvents, of which $R_{\mathrm{B}}$ is higher than the impedance of $R_{\mathrm{CT}}$ and $Z_{\mathrm{W}}$, the electric field is directly applied to $R_{\mathrm{B}}$ and $C_{\mathrm{B}}$ between the cantilever part and sample, therefore the unexpected voltage drop in the bulk solution can cause a significant electrostatic force on the cantilever part. In order to measure the local $F_{\text {esf }}$ and $\Delta \psi_{\mathrm{d}}$, we need to 
reduce this long-range parasitic force acting on the cantilever.

\section{CRITERIA FOR NANO-SCALE LOCAL SURFACE POTENTIAL MEASUREMENT}

In Sec. V, we showed that reducing the long-range parasitic force acting on the cantilever is essential for local surface potential measurement. In this section, we show the experimental results on the local electrostatic force measurements using a colloidal probe cantilever expecting the reduction of the long-range parasitic force. Then we empirically derive the criteria for the geometry of the cantilever and tip with which the nano-scale local surface charge or potential measurement can be achieved.

\section{A. Comparison between normal cantilever and colloidal probe}

In order to increase the electrostatic force acting on the tip apex, we used a gold coated $\mathrm{CP}$ cantilever, whose details were explained in Sec. II. Figure 6(a) shows the schematics of the electric field distribution between a cantilever with a normal sharp tip and the sample in air. The electric field exists between the entire cantilever and sample. Figure 6(b) shows the electric field distribution between the normal cantilever and sample in water. Since the electric field is screened by the EDLs, there is no electric field between the cantilever and sample. Figure 6(c) shows the electric field distribution between a CP cantilever and sample in water. Since the effective interaction area of the $\mathrm{CP}$ is much larger than that of the normal cantilever, it is expected that we can detect a much stronger signal by using the $\mathrm{CP}$ cantilever than the normal cantilever.

We used the second resonance mode of the cantilever, which was around $855 \mathrm{kHz}$, in order to excite the cantilever at a high frequency. Figure 7(a) shows plot of the amplitude of the $1 \omega_{\mathrm{m}}$ component as a function of $V_{\mathrm{dc}}$, obtained when the tip-sample distance was kept at about $10 \mathrm{~nm}$, and $V_{\mathrm{dc}}$ and $V_{\text {ac }}$ were set at $0 \mathrm{~V}$ and $2.8 \mathrm{~V}$ peak-to-peak, respectively. This experiment was conducted in pure water. The result shows that the hysteresis caused by the surface stress effect is negligible, but that there is no minimum point in this measurement range. This fact means that the KFM bias voltage feedback cannot be used for the surface potential measurement. As explained in Fig. 5(e), $V_{\mathrm{dc}}$ is mainly applied to $C_{\mathrm{S}}$, and partially applied to $C_{\mathrm{B}}$. The charge induced in $C_{\mathrm{S}}$ causes the surface stress change, but only $C_{\mathrm{S}}$ on the cantilever excites the cantilever oscillation, while $C_{\mathrm{S}}$ on the probe of the sphere does not excite the oscillation. Therefore, only the electric field in $C_{\mathrm{B}}$ causes the effective force on the probe of the sphere. The amplitude in Fig. 7(a) decreases with an increase in $V_{\mathrm{dc}}$, thereby the force minimum might be observed in the range of much higher than $1 \mathrm{~V}$. However, such a high $V_{\mathrm{dc}}$ should not be used because any further increase in $V_{\mathrm{dc}}$ causes the electrolysis and/or undesired electrochemical reactions. Depending on the combination of the materials of the tip and sample and the electrolyte solution, the force minimum might be observed in the measurement range, but it is difficult to estimate the surface potential difference, since most of $V_{\mathrm{dc}}$ drops in $C_{\mathrm{S}}$ especially in strong electrolytes. This result suggests that the open-loop method with a consideration of the voltage division might be an effective strategy for the surface potential measurement in aqueous solutions. Next, we discuss the tip-sample distance dependence of the electrostatic force in order to discuss the possibility of the local surface potential measurement.

Figures 7(b) and 7(c) show plots of the amplitudes of the $1 \omega_{\mathrm{m}}$ and $2 \omega_{\mathrm{m}}$ components as a function of the tip-sample distance, respectively. In both results, as the tip-sample distance was decreased, the magnitude of both components increased due to the increase in $C_{\mathrm{B}}$. The purple broken line shows the offset caused by the electrostatic force acting on the cantilever, which has almost no dependence on the tipsample distance. Comparing the $1 \omega_{\mathrm{m}}$ and $2 \omega_{\mathrm{m}}$ components, the increase in the $2 \omega_{\mathrm{m}}$ component was almost double the offset at the closest distance, while $1 \omega_{\mathrm{m}}$ component showed a steep increase. This is because $V_{\mathrm{dc}}$ is screened by $C_{\mathrm{S}}$ on both the cantilever and sample surfaces. This result suggests that the measurement of the $1 \omega_{\mathrm{m}}$ component produces a spatial resolution higher than that for the $2 \omega_{\mathrm{m}}$ component because of the EDL screening effect. Note that the electrostatic force of the $1 \omega_{\mathrm{m}}$ component acting on the cantilever cannot be explained by the GCS theory, and this originates from the voltage division between $R_{\mathrm{CT}}$ and $R_{\mathrm{B}}$ as already mentioned.

The blue and green broken curves shown in Figs. 7(b) and 7 (c) are the fitting curves calculated by the theoretical equations (see Eqs. (B13) and (B14) in Appendix B) without (a) normal tip in air

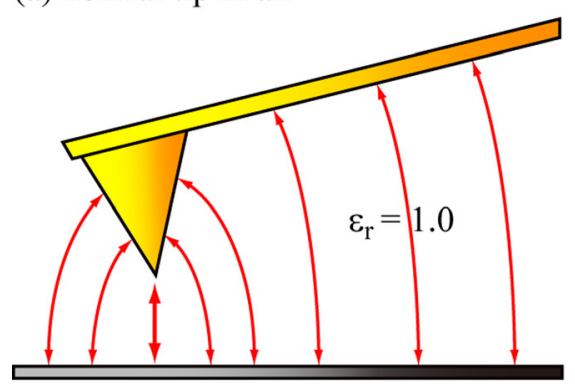

(b) normal tip in water

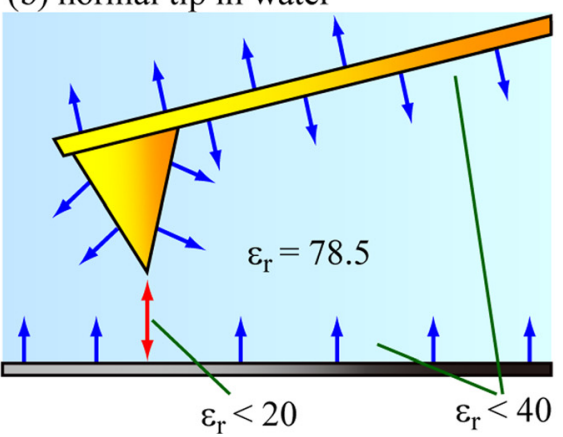

(c) colloidal probe in water

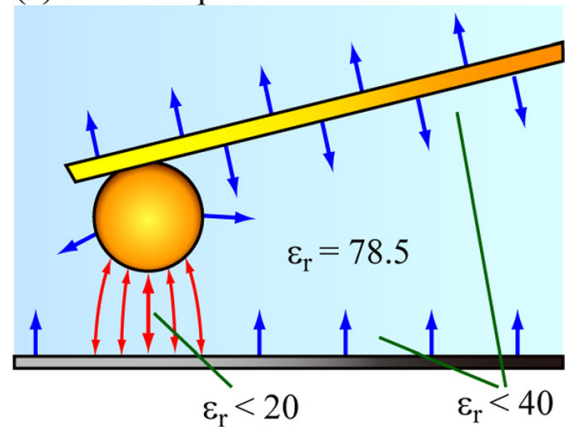

FIG. 6. Schematics of the electric field distributions in the experimental conditions with a metal coated normal cantilever in (a) air, (b) water, and (c) a metal coated CP cantilever in water. The red arrows show the electric field between the tip and sample, and the blue arrows show the electric field in the EDLs. 
(a)

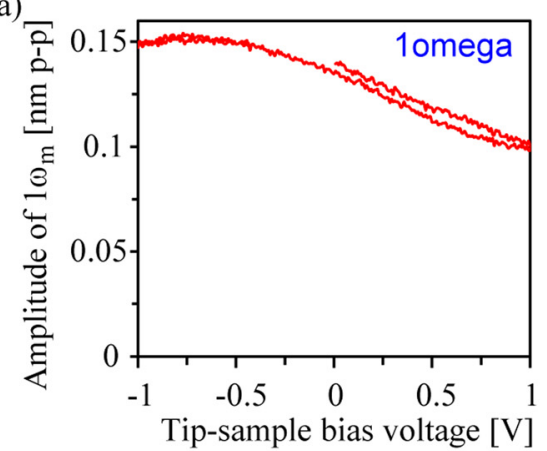

(b)

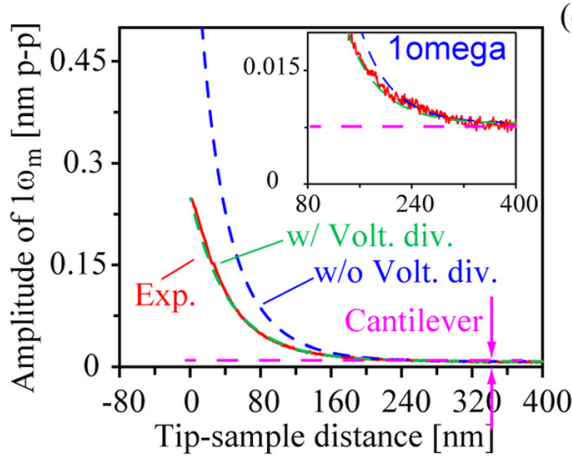

(c)

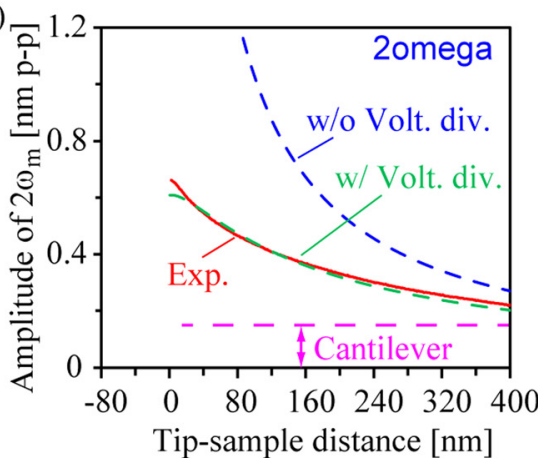

FIG. 7. Plots of amplitude of (a) $1 \omega_{\mathrm{m}}$ as a function of $V_{\mathrm{dc}}$ in water. Plots of amplitudes of (b) $1 \omega_{\mathrm{m}}$ component $\left(f_{\mathrm{m}}=f_{2}=855 \mathrm{kHz}\right)$ and (c) $2 \omega_{\mathrm{m}}$ component $\left(f_{\mathrm{m}}=f_{2} / 2=428 \mathrm{kHz}\right)$ in cantilever deflection signal as a function of the tip-sample distance in water. The inset in (b) shows the magnified data at the large distance. The red, blue, and green curves show the experimental curve, theoretically fitted curve without voltage division, and with voltage division, respectively. The purple broken line shows the offset in the oscillation amplitude caused by the electrostatic/capacitive force acting on the cantilever other than the colloid.

and with the voltage division by $C_{\mathrm{S}}$, respectively. It is difficult to accurately estimate the sensitivity of the optical deflection sensor for a higher order resonance-mode of the cantilever. Therefore, we used the amplitude of the $2 \omega_{\mathrm{m}}$ component as the reference value, whose signal is not dependent on $V_{\mathrm{dc}}$. However, there is significant disagreement between the experimental and theoretical curves without the voltage division, especially in the $2 \omega_{\mathrm{m}}$ component. The voltage division between $C_{\mathrm{B}}$ and $C_{\mathrm{S}}$ as well as that between $C_{\mathrm{B}}$ and the adsorbates and/or contaminants on the surfaces of the tip and sample significantly reduces the electrostatic force because the dielectric constant of the bulk-solution is much higher than most of the solid species. Furthermore, the reduction of the dielectric constant in the EDL by the electric field formed by the surface charge should be considered. This phenomenon is known as the dielectric saturation phenomenon. ${ }^{55-59}$ The electrostatic force at the tip-sample distance of less than $30 \mathrm{~nm}$ is affected by the dielectric saturation due to the high ac electric field. Based on the Brownian motion noise measurement, $k_{z}$ and $Q$ of the second resonance of the $\mathrm{CP}$ cantilever were determined to be $1350 \mathrm{~N} / \mathrm{m}$ and 12, respectively. The best fitted values of the local surface potential difference on the tip apex $\Delta \psi_{\mathrm{d} \_ \text {local }}$, the parasitic surface potential difference on the entire cantilever $\Delta \psi_{\mathrm{d} \_ \text {para }}{ }^{60}{ }^{6} \lambda_{\mathrm{D}}$, and $\delta C_{\mathrm{S}}$ were $0.25 \mathrm{~V}, 0.035 \mathrm{~V}, 30 \mathrm{~nm}$, and $0.011 \mathrm{~F} / \mathrm{m}^{2}$, respectively. $\lambda_{\mathrm{D}}$ estimated from the experiment was smaller than $200 \mathrm{~nm}$, which is theoretically predicted from the electrolytic concentration of pure water with dissolved $\mathrm{CO}_{2}$ gas. This inconsistency can be attributed to the fact that the solution is contaminated by some impurities. $\delta C_{\mathrm{S}}$ was also smaller than $0.2-0.3 \mathrm{~F} / \mathrm{m}^{2}$, which is typically measured by the mercury electrode without the roughness factor. This inconsistency can also be attributed to the voltage division at the surface adsorbates or contaminants.

\section{B. Criteria for geometry of cantilever and tip}

The experimental results presented above suggest that the quantitative surface potential measurement can be made by the open-loop method. Finally, we discuss the criteria for the geometry of the cantilever and tip required for the local surface potential measurements. The amplitude of the $1 \omega_{\mathrm{m}}$ component acting on the tip (Signal) is proportional to $R_{\text {tip }}$ and $\Delta \psi_{\mathrm{d} \_ \text {local }}$, therefore the Signal is given by

$$
\text { Signal } \propto \frac{Q}{k_{z}} R_{\text {tip }} \Delta \psi_{\text {d_local }} .
$$

The amplitude of the $1 \omega_{\mathrm{m}}$ component acting on the tip (Background) is proportional to $l, w$, the inverse of $h$, and $\Delta \psi_{\mathrm{d} \_ \text {para }}$, therefore the Background is given by

$$
\text { Background } \propto \frac{Q}{k_{z}} \frac{l w \Delta \psi_{\mathrm{d} \_ \text {para }}}{h},
$$

and the Signal to Background (S/B) ratio is given by

$$
\mathrm{S} / \mathrm{B}=a \frac{R_{\text {tip }} h}{l w} \frac{\Delta \psi_{\text {d_local }}}{\Delta \psi_{\text {d_para }}},
$$

where $a$ is a complicated function of voltage division and dielectric saturation, but can be assumed to be a constant when the parameters other than the geometry of the cantilever and tip are fixed. In Fig. 7(b), the amplitude of the $1 \omega_{\mathrm{m}}$ component acting on the cantilever is $0.008 \mathrm{~nm}$, which is on the same order as that of the result of Fig. 3(c) in water because both cantilevers have the same nominal spring constant. On the other hand, the amplitude of the $1 \omega_{\mathrm{m}}$ component acting on the cantilever is $0.5 \mathrm{~nm}$, and the $\mathrm{S} / \mathrm{B}$ ratio is estimated to about 50. Substituting the parameters of the $\mathrm{CP}$ cantilever $\left(h=2 R_{\text {tip }}\right)$ and the value of the $\mathrm{S} / \mathrm{B}$ ratio $(=50)$ into Eq. (19) gives $a$ as 1200. This equation means that reducing the dimensions of the cantilever and increasing the tip height enable us to suppress the parasitic oscillation caused by the long-range electrostatic force. Reducing the dimensions of the cantilever is more preferred than enlarging the tip height, because this also leads to a cantilever with a high resonance frequency and a low spring constant which enables the force detection with a high signal-to-noise ratio. Concurrently, $R_{\text {tip }}$ should be as small as possible in order to keep the high spatial resolution. Figure 8 shows the relationship between $R_{\text {tip }}$ and calculated $\mathrm{S} / \mathrm{B}$ values of the commercially available cantilevers. This calculation was conducted under the condition that $R_{\text {tip }}$ of the metal-coated nanoscopic tip is $30 \mathrm{~nm}$, and $\Delta \psi_{\mathrm{d} \_ \text {local }}, \Delta \psi_{\mathrm{d} \_ \text {para }}$, are the same as that 


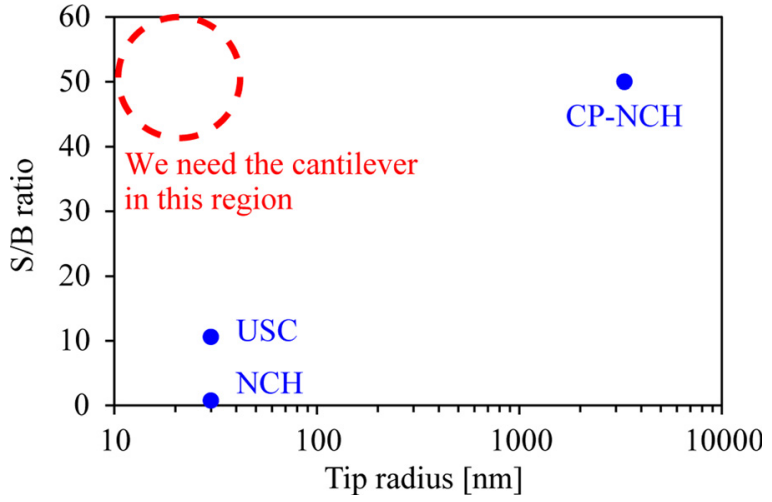

FIG. 8. Relationship between tip radius and S/B ratio. The cantilever parameters of $\mathrm{NCH}$ and $\mathrm{CP}-\mathrm{NCH}$ were given by the datasheet, and that of USC was given by the SEM measurement of Ref. 61.

obtained in the $\mathrm{CP}$ measurement. The $\mathrm{S} / \mathrm{B}$ ratio of the PPP$\mathrm{NCH}$ cantilever is estimated to be only 0.75 . This means that the surface area of the cantilever should be minimized to 1/67 for obtaining the same $\mathrm{S} / \mathrm{B}$ ratio as that of the $\mathrm{CP}$ cantilever. An ultrashort cantilever (Nanoworld: USC), which was developed for high-speed AFM applications, is also compared with the other cantilevers. Although this cantilever is much smaller than PPP-NCH, its tip height is much smaller than those of the other normal cantilevers, thereby the $\mathrm{S} / \mathrm{B}$ ration does not increase much. It is necessary to develop a small cantilever with a high aspect ratio tip for the nano-scale local potential measurements. Note that even if such a dedicated force sensor is available, the Stern layer capacitance exists on all of the material surfaces. Furthermore, the dielectric constants of typical dielectric samples, such as biomolecules, organic molecules, and colloidal spheres, are significantly lower than that of water. In such a case, the modulation voltage strongly attenuates in the sample, therefore great care should be taken to estimate the local surface potential on these samples.

\section{CONCLUSIONS}

We measured the electrostatic and capacitive forces induced on a conductive cantilever with a tip when an alternating voltage is applied between the cantilever and a sample surface in various liquids including an aqueous solution. We found that it is possible to measure the local surface potential distribution by the KFM technique in air and non-polar medium, but it is not possible to perform the same measurement using the conventional schemes in aqueous solution. In aqueous solution, the cantilever deflection is predominantly caused by the surface stress especially when the modulation frequency is low and that the electrostatic force contribution to the cantilever deflection becomes dominant in a high frequency range which is typically higher than tens of $\mathrm{kHz}$. However, we could not obtain the steep increase in the electrostatic force acting on the tip apex near the sample surface and also could not obtain reproducible result regarding the dependence of the electrostatic force on the dc bias voltage. From the simple theoretical predictions, as the tip-sample distance is decreased, the bulk solution capacitance is increased, and the electrostatic force should be observed even in the aqueous solution. We conducted the experiment using a CP cantilever and obtained good agreement with the theoretical calculation considering the voltage division and the dielectric saturation. This result suggests that the effective interaction area of the normal cantilever is much smaller than the area of the entire cantilever, and the long-range parasitic electrostatic force as well as the surface stress effect is dominant. For the local surface potential measurement, a cantilever with small surface area and/or the high tip height is required. Reducing the dimensions of the cantilever also enables to maintaining a sufficient signal intensity even at a high frequency range. Even if the local information on the surface potential can be detected, the KFM bias feedback cannot be used because the voltage drop in the Stern layer is dominant. The open-loop method with the force mapping technique and careful treatment of several factors related to the EDLs are required for the quantitative surface potential measurements.

\section{ACKNOWLEDGMENTS}

This work was supported by a Grant-in-Aid for Scientific Research from the Ministry of Education, Culture, Sports, Science and Technology of Japan, SENTAN Program of the Japan Science and Technology Agency. The authors would also like to thank Ryohei Kokawa, Masahiro Ohta, and Kazuyuki Watanabe of Shimadzu Corporation.

\section{APPENDIX A: CALCULATION OF SURFACE STRESS CONTRIBUTION}

Surface charge density $\sigma_{\mathrm{EDL}}$ accumulated in $C_{\mathrm{EDL}}$ is given by integration of capacitance per unit area by $\psi$, and expressed as

$$
\sigma_{\mathrm{EDL}}=\int_{0}^{\psi_{0}} \delta C_{\mathrm{EDL}} \mathrm{d} \psi
$$

where $\delta C_{\mathrm{EDL}}$ is $C_{\mathrm{EDL}}$ per unit area, which is a serial capacitance of $C_{\mathrm{D}}$ per unit area $\delta C_{\mathrm{D}}$ and $C_{\mathrm{S}}$ per unit area $\delta C_{\mathrm{S}}$, and expressed as

$$
\begin{aligned}
\delta C_{\mathrm{EDL}} & =\frac{1}{\delta C_{\mathrm{S}}^{-1}+\delta C_{\mathrm{D}}^{-1}} \\
& =\frac{1}{\delta \eta_{\mathrm{S}} \delta C_{\mathrm{S}}^{-1}+\left[\delta \eta_{\mathrm{D}} \varepsilon_{0} \varepsilon_{\mathrm{r}} \kappa_{\mathrm{D}} \cosh \left(\frac{e \psi_{\mathrm{D}}}{2 k_{\mathrm{B}} T}\right)\right]^{-1}},
\end{aligned}
$$

where $\eta_{\mathrm{S}}$ and $\eta_{\mathrm{D}}$ are roughness factor of the Stern layer and the diffuse layer, which varies depending on $\lambda_{\mathrm{D}}$, respectively. These roughness factors are 1 for the liquid mercury electrode, but the polycrystalline electrode, which is used for the cantilever metal coating, is around 3 depending on the surface morphology. ${ }^{62}$ Surface energy $\gamma_{\text {ss }}$ is given by double integration of $\delta C_{\mathrm{EDL}}$, expressed as ${ }^{44}$

$$
\gamma_{\mathrm{ss}}=\int_{0}^{\psi_{0}} q^{\mathrm{M}} \mathrm{d} \psi=\gamma_{\mathrm{pzc}}-\iint_{0}^{\psi_{0}} \delta C_{\mathrm{EDL}} \mathrm{d} \psi^{2}
$$


Since $\delta C_{\mathrm{S}}$ is not a function of potential but $\delta C_{\mathrm{D}}$ is a function of $\psi_{\mathrm{D}}$, much high modulation voltage is not applied to $\delta C_{\mathrm{D}}$. But practically, average constant $\delta C_{\mathrm{D}}$ is effective at the frequency higher than tens of $\mathrm{kHz}$. Therefore, $\gamma_{\mathrm{ss}}$ caused by modulation potential $\psi_{\mathrm{ac}}=\psi_{0}+\psi_{0 \_\mathrm{ac}} \cos \omega_{\mathrm{m}} \mathrm{t}$ is expressed as

$$
\begin{aligned}
\gamma_{\mathrm{ss}}= & \gamma_{\mathrm{pzc}}-\frac{1}{2} \delta C_{\mathrm{EDL}} \psi_{\mathrm{ac}}^{2} \\
\approx & \gamma_{\mathrm{pzc}}-\delta C_{\mathrm{EDL}}\left(\frac{1}{2} \psi_{0}^{2}+\frac{1}{16} V_{\mathrm{ac}}^{2}+\frac{1}{2} \psi_{0} V_{\mathrm{ac}} \cos \omega_{\mathrm{m}} t\right. \\
& \left.+\frac{1}{16} V_{\mathrm{ac}}^{2} \cos 2 \omega_{\mathrm{m}} t\right)
\end{aligned}
$$

where $\psi_{0 \_ \text {ac }}=1 / 2 V_{\mathrm{ac}}$ is used by regarding that $\delta C_{\mathrm{EDL}}$ of the cantilever is same as $\delta C_{\mathrm{EDL}}$ of a sample. The relationship between $\gamma_{\mathrm{ss}}$ and the surface stress $\sigma_{\mathrm{ss}}$ acting on an electrode is described by the Shuttleworth equation, expressed as ${ }^{63}$

$$
\sigma_{\mathrm{ss}}=\gamma_{\mathrm{ss}}+\frac{\mathrm{d} \gamma_{\mathrm{ss}}}{\mathrm{d} \varepsilon} \approx \gamma_{\mathrm{ss}}
$$

where $\varepsilon$ is surface area change normalized by surface area. In the case of the cantilever, since the second term is much less than the first term, it can be ignored. Although $\gamma_{\mathrm{ss}}$ actually acts not only on the cantilever but also on the tip part, the effect on the tip is negligible because the thickness of the tip is much larger than that of the cantilever. Therefore, only $\gamma_{\mathrm{ss}}$ acting on the cantilever should be considered. When the cantilever is located near the sample surface, most of the ac voltage is applied on the front side of the cantilever so that only the front side of cantilever should be considered $\left(\Delta \sigma=-\sigma_{\text {front }}\right)$. By regarding the surface stress as a concentrated load acting on the end of the cantilever, the force induced by surface stress $F_{\mathrm{ss}}$ is expressed as ${ }^{64}$

$$
\begin{aligned}
F_{\mathrm{ss}}= & \frac{3(1-\nu)}{4} \frac{w t}{l} \Delta \sigma \\
\approx & -\frac{3(1-\nu)}{4} \frac{w t}{l} \delta C_{\mathrm{EDL}}\left(\frac{1}{2} \psi_{0}^{2}+\frac{1}{16} V_{\mathrm{ac}}^{2}\right. \\
& \left.+\frac{1}{2} \psi_{0} V_{\mathrm{ac}} \cos \omega_{\mathrm{m}} t+\frac{1}{16} V_{\mathrm{ac}}^{2} \cos 2 \omega_{\mathrm{m}} t\right),
\end{aligned}
$$

where $t$ is thickness of a cantilever, $\nu$ is the Poisson's ratio of the cantilever material, and $\Delta \sigma$ is the surface stress difference of the back-side and front-side of the cantilever. This equation means that the amplitude and phase of $1 \omega_{\mathrm{m}}$ component of the surface stress depend on the surface potential of cantilever, while the amplitude of $2 \omega_{\mathrm{m}}$ component depends only on $V_{\mathrm{ac}}$, and the phase of $2 \omega_{\mathrm{m}}$ component is always inphase. It is clear from Eq. (1) that the phase of $1 \omega_{\mathrm{m}}$ component of the electrostatic force depends on the surface potential of the cantilever, while the phase of $2 \omega_{\mathrm{m}}$ component is always out-of-phase because $\mathrm{d} C / \mathrm{d} z$ is always negative.

\section{APPENDIX B: FREQUENCY RESPONSE OF ELECTRIC DOUBLE LAYER FORCE FOR PLANAR SURFACES}

The metallic surfaces have the charge boundary condition near the constant potential, especially in weak electrolyte solutions such as pure water. ${ }^{52}$ For considering the voltage division effect, an analytical solution expressing the relationship of $d$, $\psi$, and $E$ is required. However, the analytical solution of P-B equation is obtainable only in the case $\psi$ is less than $0.025 \mathrm{~V}$ and the distance between the surfaces is larger than $\lambda_{\mathrm{D}} \cdot{ }^{65} \mathrm{In}$ reality, higher voltage is usually applied in many cases. For solving this problem, we made an approximate equation, which covers the all magnitude of voltage, by modifying the linear superposition approximation (LSA) ${ }^{66}$ The dc electric field at the mid-point between the surfaces is expressed as

$$
E_{\mathrm{dc}}=-\frac{\mathrm{d} \psi_{\mathrm{dc}}(d / 2)}{\mathrm{d} x}=\frac{2 k_{\mathrm{B}} T}{e}\left\{\begin{array}{l}
\frac{1}{d}\left[-\ln \left[\frac{1+\gamma_{1} \exp \left(-\kappa_{\mathrm{D}} d / 2\right)}{1-\gamma_{1} \exp \left(-\kappa_{\mathrm{D}} d / 2\right)}\right]+\ln \left[\frac{1+\gamma_{2} \exp \left(-\kappa_{\mathrm{D}} d / 2\right)}{1-\gamma_{2} \exp \left(-\kappa_{\mathrm{D}} d / 2\right)}\right]\right] \\
+2 \kappa_{\mathrm{D}}\left(-\gamma_{1}+\gamma_{2}\right) \exp \left(-\kappa_{\mathrm{D}} d / 2\right)
\end{array}\right\}
$$

where $\gamma_{i}$ is given by

$$
\gamma_{i}=\tanh \left(\frac{e \psi_{0-i}}{4 k_{\mathrm{B}} T}\right) .
$$

The first and second terms in the curly bracket of Eq. (B1) are the electric field when the EDLs of surfaces are not overlapped and are overlapped, respectively. When the distance between surfaces is smaller than $\lambda_{\mathrm{D}}$, the first term becomes dominant. When the distance between surfaces is larger than $\lambda_{\mathrm{D}}$, the second term becomes dominant. Figure 9 shows the comparison between the exact solution of the nonlinear P-B equation obtained by numerically calculating the fourthorder Runge-Kutta method and the calculated result obtained by the above equation. This shows that less than $25 \%$ error at all the distance at arbitrary voltages and the arbitrary $\lambda_{\mathrm{D}}$. The ac electric field at the mid-point between the surfaces is expressed as

$$
E_{\mathrm{ac}}=-\frac{\mathrm{d} \psi_{\mathrm{ac}}(x=d / 2)}{\mathrm{d} x}=\xi \frac{V_{\mathrm{ac}}}{d} \cos \omega_{\mathrm{m}} t,
$$

where $\xi$ is the voltage division factor, which is expressed as

$$
\xi=\frac{1+2 \frac{\delta C_{\mathrm{B}}}{\delta C_{\mathrm{s}}}}{\left(2 \frac{\delta C_{\mathrm{B}}}{\delta C_{\mathrm{s}}} \frac{\omega_{\mathrm{c}}}{\omega}\right)^{2}+\left(1+2 \frac{\delta C_{\mathrm{B}}}{\delta C_{\mathrm{s}}}\right)^{2}},
$$

where bulk solution capacitance per unit area is obtained by 


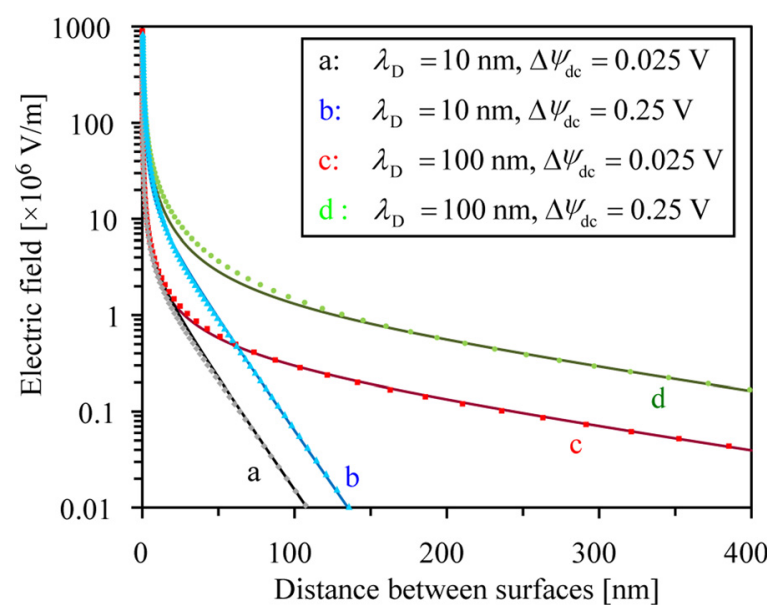

FIG. 9. Comparison of numerically calculated results (symbols) with semiempirical results (curves).

$$
\delta C_{\mathrm{B}}=\frac{\varepsilon_{0} \varepsilon_{\mathrm{r}}}{d}
$$

Furthermore, the dielectric saturation should be taken into account for the precise analysis of the electrostatic force in polar-liquids, since high ac electric field applied between the tip and sample causes the significant decrease in the orientation degree of the solvent molecules. The Booth equation is generally used for the dielectric saturation of pure water, ${ }^{55,67}$ and is expressed as

$$
\begin{aligned}
\varepsilon_{\mathrm{r}}(E) & =n_{\mathrm{w}}{ }^{2}+\left(\varepsilon_{\mathrm{r}_{-} \infty}-n_{\mathrm{w}}{ }^{2}\right) \frac{3}{\beta E} \mathcal{L}(\beta E) \\
& =n_{\mathrm{w}}{ }^{2}+\left(\varepsilon_{\mathrm{r}_{-} \infty}-n_{\mathrm{w}}{ }^{2}\right) \frac{3}{\beta E}\left[\operatorname{coth}(\beta E)-\frac{1}{\beta E}\right],
\end{aligned}
$$

where $n_{\mathrm{w}}$ is the reflective index of water, $\mathcal{L}()$ is the Langevin function, and

$$
\beta=\frac{5 \mu_{\mathrm{w}}\left(n_{\mathrm{w}}^{2}+2\right)}{2 k_{\mathrm{B}} T} \approx 1.416 \times 10^{-8} \mathrm{~m} \mathrm{~V}^{-1}
$$

where $\mu_{\mathrm{w}}$ is the electric dipole moment of water molecule. The dielectric constant is a nonlinear function of electric field but it can be approximated to a parabolic function especially below the electric field of $0.1 \mathrm{~V} / \mathrm{nm}$, expressed as

$$
\varepsilon_{\mathrm{r}}(E) \approx \varepsilon_{\mathrm{r}_{-} \infty}-v_{2} E^{2}
$$

where $v_{2}$ was estimated to $8 \times 10^{-16} \mathrm{~m}^{2} / \mathrm{V}^{2}$ by fitting the above equation to Eq. (B6). The electrostatic force term can be expressed as

$$
\begin{aligned}
& T_{\text {esf }}=-\frac{1}{2} \varepsilon_{0}\left[\varepsilon_{\mathrm{r}_{-} \infty}-v_{2} E^{2}\right] E^{2}=-\frac{1}{2} \varepsilon_{0}\left[\varepsilon_{\mathrm{r}_{-} \infty} E^{2}-v_{2} E^{4}\right] \\
& =-\frac{1}{2} \varepsilon_{0}\left\{\begin{array}{c}
\varepsilon_{\mathrm{r}_{-} \infty}\left[\begin{array}{l}
E_{\mathrm{dc}}{ }^{2}+\frac{1}{2} E_{\mathrm{ac}}^{2}+2 E_{\mathrm{dc}} E_{\mathrm{ac}} \cos \omega_{\mathrm{m}} t+\frac{1}{2} E_{\mathrm{ac}}^{2} \cos 2 \omega_{\mathrm{m}} t
\end{array}\right] \\
-v_{2}\left[\begin{array}{l}
E_{\mathrm{dc}}{ }^{4}+\frac{3}{8} E_{\mathrm{ac}}^{4}+3 E_{\mathrm{dc}}^{2} E_{\mathrm{ac}}^{2} \\
+\left(4 E_{\mathrm{dc}}^{3} E_{\mathrm{ac}}+3 E_{\mathrm{dc}} E_{\mathrm{ac}}{ }^{3}\right) \cos \omega_{\mathrm{m}} t \\
+\left(3 E_{\mathrm{dc}}^{2} E_{\mathrm{ac}}^{2}+\frac{1}{2} E_{\mathrm{ac}}^{4}\right) \cos 2 \omega_{\mathrm{m}} t \\
+E_{\mathrm{dc}} E_{\mathrm{ac}}^{3} \cos 3 \omega_{\mathrm{m}} t+\frac{1}{8} E_{\mathrm{ac}}^{4} \cos 4 \omega_{\mathrm{m}} t
\end{array}\right]
\end{array}\right\} .
\end{aligned}
$$

These equations can be used for the calculation of the electrostatic force depending on the modulation frequency. The electrostatic force with the dielectric saturation is half the value of that without the dielectric saturation in the case that the tip-sample distance is about $10 \mathrm{~nm}$ and $V_{\text {ac }}$ is $2.8 \mathrm{~V}$ peakto-peak. Note that even in the case when the non-linearity of the dielectric constant of medium is considered, $1 \omega_{\mathrm{m}}$ component is observed only when $E_{\mathrm{dc}}$ exists.

Figures 10(a) and 10(b) show the electrostatic force per unit area of the $1 \omega_{\mathrm{m}}$ and $2 \omega_{\mathrm{m}}$ components as a function of the tip-sample distance, respectively. The calculation was done under $V_{\mathrm{dc}}=0.1 \mathrm{~V}, \quad V_{\mathrm{ac}}=2.8 \mathrm{~V}$ peak-to-peak, $\lambda_{\mathrm{D}}=30 \mathrm{~nm}$, of which corresponds to $0.1 \mathrm{mM}$ solution, and $\delta C_{\mathrm{S}}=0.03 \mathrm{~F} / \mathrm{m}^{2}$. The numbers shown in the legends are the ratio of $f_{\mathrm{m}} / f_{\mathrm{c}}$, and the calculation result without the EDL is also shown in same figure. In both the $1 \omega_{\mathrm{m}}$ and $2 \omega_{\mathrm{m}}$ components, the electrostatic force increase as a modulation frequency increases. The absolute quantity of the $2 \omega_{\mathrm{m}}$ component is higher than that of the $1 \omega_{\mathrm{m}}$ component, but the $2 \omega_{\mathrm{m}}$ component significantly decreases as the modulation frequency decreases. The $1 \omega_{\mathrm{m}}$ component is more strongly affected by the EDL screening effect than the $2 \omega_{\mathrm{m}}$ component, while the $2 \omega_{\mathrm{m}}$ component is more strongly affected by the voltage division effect than the $1 \omega_{\mathrm{m}}$ component. At the frequency of infinity, the electrostatic force of the $2 \omega_{\mathrm{m}}$ component is almost the same as that without EDL screening effect at the distance of larger than $\lambda_{\mathrm{D}}$, but show significant discrepancy at the distance of smaller than $\lambda_{\mathrm{D}}$. On the other hand, the electrostatic force of the $1 \omega_{\mathrm{m}}$ component is almost the same as that without the EDL screening effect at the 
(a)
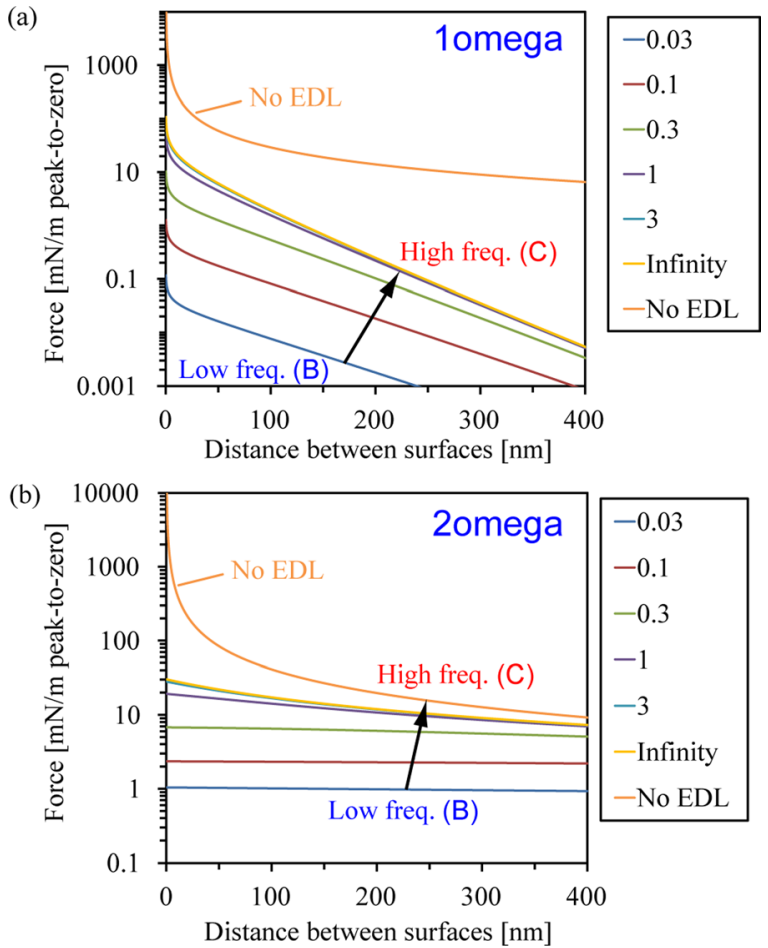

FIG. 10. Plots of electrostatic force per unit area of (a) $1 \omega_{\mathrm{m}}$, and (b) $2 \omega_{\mathrm{m}}$ components as a function of the tip-sample distance. The values in the legend show the ratio of $f_{\mathrm{m}} / f_{\mathrm{c}}$ considering voltage division by the EDLs. Infinity means that $f_{\mathrm{m}} / f_{\mathrm{c}}$ is infinity value. No EDL means that voltage division by the EDLs is not considered.

distance of smaller than $\lambda_{\mathrm{D}}$, but show significant discrepancy at the distance of larger than $\lambda_{\mathrm{D}}$. These calculations show that only the apex of the tip should be considered for the $1 \omega_{\mathrm{m}}$ component, and the entire cantilever should be considered for the $2 \omega_{\mathrm{m}}$ component.

For the calculation of the theoretical curve in Fig. 7(c), we summed the electrostatic force of sphere $F_{\text {sphere }}$ and $F_{\mathrm{cl}}$ as

$$
F_{\text {esf }}=F_{\text {sphere }}+F_{\text {cl }},
$$

where $F_{\text {sphere }}$ is obtained by integrating $E$ over the surface by the equation expressed as ${ }^{68}$

$$
\begin{aligned}
F_{\text {sphere }} & =\int_{S} T_{\text {esf }}\left(l^{\prime}, \omega_{\mathrm{m}}\right) \mathrm{d} S \mathbf{n} \cdot \mathbf{u}_{\mathbf{z}} \\
& =2 \pi R_{\text {apex }}{ }^{2} \int_{0}^{\pi} T_{\text {esf }}\left(l^{\prime}, \omega_{\mathrm{m}}\right) \theta \mathrm{d} \theta,
\end{aligned}
$$

where $l^{\prime}$ is the tip-sample distance at each part of the tip expressed as

$$
l^{\prime}=\frac{\theta\left[z+z_{0}+R_{\text {apex }}(1-\cos \theta)\right]}{\sin \theta},
$$

where $z$ is the height of the nanoscopic tip above the surface and $z_{0}$ is the difference between the mesoscopic and the nanoscopic tip heights. We used $10 \mathrm{~nm}$ for $z_{0}$ for the calculation, which was the value typically obtained by the EDL force measurement. The oscillation amplitude is obtained by

$$
A_{\text {esf }}=\frac{Q_{2 \text { nd }} F_{\text {esf }}}{k_{z \_2 \text { nd }}} .
$$

Considering the squeeze damping effect, the net value of oscillation amplitude is obtained by

$$
A_{\text {esf }}{ }^{\prime}=A_{\text {esf }}\left(\frac{2 \pi f_{2} Q_{\infty} \_ \text {nd }}{k_{z \_2 \text { nd }}} \frac{6 \pi \eta R_{\text {tip }}^{2}}{z+z_{0}}+1\right)^{-1} .
$$

${ }^{1}$ Y. Martin, D. W. Abraham, and H. K. Wickramasinghe, Appl. Phys. Lett. 52, 1103 (1988).

${ }^{2}$ J. E. Stern, B. D. Terris, H. J. Mamin, and D. Rugar, Appl. Phys. Lett. 53, 2717 (1988).

${ }^{3}$ M. Nonnenmacher, M. P. O'Boyle, and H. K. Wickramasinghe, Appl. Phys. Lett. 58, 2921 (1991).

${ }^{4}$ J. M. R. Weaver and D. W. Abraham, J. Vac. Sci. Technol., B 9, 1559 (1991).

${ }^{5}$ A. L. Domanski, E. Sengupta, K. Bley, M. B. Untch, S. A. L. Weber, K. Landfester, C. K. Weiss, H. J. Butt, and R. Berger, Langmuir 28, 13892 (2012).

${ }^{6}$ B. W. Hoogenboom, H. J. Hug, Y. Pellmont, S. Martin, P. L. T. M. Frederix, D. Fotiadis, and A. Engel, Appl. Phys. Lett. 88, 193109 (2006).

${ }^{7}$ T. Ando, T. Uchihashi, and T. Fukuma, Prog. Surf. Sci. 83, 337 (2008).

${ }^{8}$ H. Yamada, K. Kobayashi, T. Fukuma, Y. Hirata, T. Kajita, and K. Matsushige, Appl. Phys. Express 2, 095007 (2009).

${ }^{9}$ L. Collins, J. I. Kilpatrick, I. V. Vlassiouk, A. Tselev, S. A. L. Weber, S. Jesse, S. V. Kalinin, and B. J. Rodriguez, Appl. Phys. Lett. 104, 133103 (2014).

${ }^{10}$ S. Banerjee, J. Shim, J. Rivera, X. Z. Jin, D. Estrada, V. Solovyeva, X. You, J. Pak, E. Pop, N. Aluru, and R. Bashir, ACS Nano 7, 834 (2013).

${ }^{11}$ X. Yin and J. Drelich, Langmuir 24, 8013 (2008).

${ }^{12}$ I. Siretanu, D. Ebeling, M. P. Andersson, S. L. S. Stipp, A. Philipse, M. C. Stuart, D. van den Ende, and F. Mugele, Sci. Rep. 4, 4956 (2014).

${ }^{13}$ K. Umeda and K. Fukui, J. Vac. Sci. Technol., B 28, C4D40 (2010).

${ }^{14}$ K. Umeda and K. Fukui, Langmuir 26, 9104 (2010).

${ }^{15}$ K. Suzuki, K. Kobayashi, N. Oyabu, K. Matsushige, and H. Yamada, J. Chem. Phys. 140, 054704 (2014).

${ }^{16}$ Y. Yang, K. M. Mayer, N. S. Wickremasinghe, and J. H. Hafner, Biophys. J. 95, 5193 (2008)

${ }^{17}$ J. Sotres, A. Lostao, C. Gómez-Moreno, and A. M. Baró, Ultramicroscopy 107, 1207 (2007).

${ }^{18}$ J. Sotres and A. M. Baró, Appl. Phys. Lett. 93, 103903 (2008).

${ }^{19}$ J. Sotres and A. M. Baró, Biophys. J. 98, 1995 (2010).

${ }^{20}$ D. Ebeling, D. van den Ende, and F. Mugele, Nanotechnology 22, 305706 (2011).

${ }^{21}$ Y. Hirata, F. Mizutani, and H. Yokoyama, Surf. Interface Anal. 27, 317 (1999).

${ }^{22}$ M. P. Scherer, G. Frank, and A. W. Gummer, J. Appl. Phys. 88, 2912 (2000).

${ }^{23}$ A. M. Hilton, B. P. Lynch, and G. J. Simpson, Anal. Chem. 77, 8008 (2005).

${ }^{24}$ B. P. Lynch, A. M. Hilton, C. H. Doerge, and G. J. Simpson, Langmuir 21, $1436(2005)$

${ }^{25}$ A. M. Hilton, B. P. Lynch, and G. J. Simpson, Am. Lab. 38, 23 (2006).

${ }^{26}$ B. P. Lynch, A. M. Hilton, and G. J. Simpson, Biophys. J. 91, 2678 (2006).

${ }^{27}$ B. J. Rodriguez, S. Jesse, K. Seal, A. P. Baddorf, and S. V. Kalinin, J. Appl. Phys. 103, 014306 (2008).

${ }^{28}$ K. Umeda, N. Oyabu, K. Kobayashi, Y. Hirata, K. Matsushige, and H. Yamada, Appl. Phys. Express 3, 065205 (2010).

${ }^{29}$ N. Kobayashi, H. Asakawa, and T. Fukuma, Rev. Sci. Instrum. 81, 123705 (2010).

${ }^{30}$ N. Kobayashi, H. Asakawa, and T. Fukuma, J. Appl. Phys. 110, 044315 (2011).

${ }^{31}$ G. Gramse, M. A. Edwards, L. Fumagalli, and G. Gomila, Appl. Phys. Lett. 101, 213108 (2012).

${ }^{32}$ N. Kobayashi, H. Asakawa, and T. Fukuma, Rev. Sci. Instrum. 83, 033709 (2012).

${ }^{33}$ G. Gramse, A. Dols-Perez, M. A. Edwards, L. Fumagalli, and G. Gomila, Biophys. J. 104, 1257 (2013).

${ }^{34}$ G. Gramse, M. A. Edwards, L. Fumagalli, and G. Gomila, Nanotechnology 24, 415709 (2013).

${ }^{35}$ B. Kumar and S. R. Crittenden, Nanotechnology 24, 435701 (2013). 
${ }^{36}$ J. J. Zhang, D. M. Czajkowsky, Y. Shen, J. L. Sun, C. H. Fan, J. Hu, and Z. F. Shao, Appl. Phys. Lett. 102, 073110 (2013).

${ }^{37}$ K. Umeda, K. Kobayashi, K. Matsushige, and H. Yamada, Appl. Phys. Lett. 101, 123112 (2012).

${ }^{38}$ K. Umeda, K. Kobayashi, N. Oyabu, Y. Hirata, K. Matsushige, and H. Yamada, J. Appl. Phys. 113, 154311 (2013).

${ }^{39}$ D. J. Marchand, E. Hsiao, and S. H. Kim, Langmuir 29, 6762 (2013).

${ }^{40}$ L. Collins, S. Jesse, J. I. Kilpatrick, A. Tselev, O. Varenyk, M. B. Okatan, S. A. L. Weber, A. Kumar, N. Balke, S. V. Kalinin, and B. J. Rodriguez, Nat. Commun. 5, 3871 (2014).

${ }^{41}$ J. E. Sader, J. W. M. Chon, and P. Mulvaney, Rev. Sci. Instrum. 70, 3967 (1999).

${ }^{42}$ H. Sugimura, Y. Ishida, K. Hayashi, O. Takai, and N. Nakagiri, Appl. Phys. Lett. 80, 1459 (2002).

${ }^{43}$ R. Raiteri and H.-J. Butt, J. Phys. Chem. 99, 15728 (1995).

${ }^{44}$ A. J. Bard and L. R. Faulkner, Electrochemical methods, Fundamentals and Applications, 2nd ed. (John Wiley \& Sons, Inc., 2001).

${ }^{45}$ J. E. B. Randles, Discuss. Faraday Soc. 1, 11 (1947).

${ }^{46} \mathrm{E}$. Barsoukov and J. R. Macdonald, Impedance Spectroscopy: Theory, Experiment, and Applications, 2nd ed. (John Wiley \& Sons, Inc., 2005).

${ }^{47}$ T. L. Sounart, T. A. Michalske, and K. R. Zavadil, J. Microelectromech. Syst. 14, 125 (2005).

${ }^{48}$ V. Mukundan, P. Ponce, H. E. Butterfield, and B. L. Pruitt, J. Micromech. Microeng. 19, 065008 (2009).

${ }^{49}$ J. F. Zhang, A. Drechsler, K. Grundke, and D. Y. Kwok, Colloids Surf., A 242, 189 (2004).

${ }^{50}$ M. Z. Bazant, K. Thornton, and A. Ajdari, Phys. Rev. E 70, 021506 (2004).

${ }^{51}$ D. Y. C. Chan and D. J. Mitchell, J. Colloid Interface Sci. 95, 193 (1983).
${ }^{52}$ R. Pericet-Camara, G. Papastavrou, S. H. Behrens, and M. Borkovec, J. Phys. Chem. B 108, 19467 (2004).

${ }^{53}$ M. V. Salapaka, H. S. Bergh, J. Lai, A. Majumdar, and E. McFarland, J. Appl. Phys. 81, 2480 (1997).

${ }^{54}$ J. E. Sader, J. Appl. Phys. 84, 64 (1998).

${ }^{55}$ F. Booth, J. Chem. Phys. 19, 391 (1951).

${ }^{56}$ V. N. Paunov, R. I. Dimova, P. A. Kralchevsky, G. Broze, and A. Mehreteab, J. Colloid Interface Sci. 182, 239 (1996).

${ }^{57}$ A. Abrashkin, D. Andelman, and H. Orland, Phys. Rev. Lett. 99, 077801 (2007).

${ }^{58}$ E. Gongadze and A. Iglič, Bioelectrochemistry 87, 199 (2012).

${ }^{59}$ A. Levy, D. Andelman, and H. Orland, Phys. Rev. Lett. 108, 227801 (2012).

${ }^{60}$ The parasitic surface potential difference may be caused by undesired electrolysis and/or electrochemical reactions. This causes the long-range parasitic electrostatic force which does not show the distance dependency.

${ }^{61}$ T. Fukuma, K. Onishi, N. Kobayashi, A. Matsuki, and H. Asakawa, Nanotechnology 23, 135706 (2012).

${ }^{62}$ J. Weissmuller and H. L. Duan, Phys. Rev. Lett. 101, 146102 (2008).

${ }^{63}$ R. Shuttleworth, Proc. Phys. Soc. London, Ser. A 63, 444 (1950).

${ }^{64}$ M. Godin, V. Tabard-Cossa, P. Grütter, and P. Williams, Appl. Phys. Lett. 79, 551 (2001).

${ }^{65}$ R. Hogg, T. W. Healy, and D. W. Fuersten, Trans. Faraday Soc. 62, 1638 (1966).

${ }^{66} \mathrm{H}$. Ohshima, Biophysical Chemistry of Biointerfaces (John Wiley \& Sons, Inc., 2010).

${ }^{67}$ L. Yang, B. H. Fishbine, A. Migliori, and L. R. Pratt, J. Chem. Phys. 132, 044701 (2010).

${ }^{68}$ S. Hudlet, M. Saint Jean, C. Guthmann, and J. Berger, Eur. Phys. J. B 2, 5 (1998). 